\title{
UNIFORM BOUNDS AND WEAK SOLUTIONS TO AN OPEN SCHRÖDINGER-POISSON SYSTEM*
}

\author{
OLIVIER PINAUD ${ }^{\dagger}$
}

\begin{abstract}
This paper is concerned with the derivation of uniform bounds with respect to the scaled Planck constant $\varepsilon$ for solutions to the open transient Schrdinger-Poisson system introduced by Ben Abdallah et al in [Math. Meth. Mod. in App. Sci., 15, 667-688, 2005]. The uniform estimates stem from a careful analysis of the non-local in time transparent boundary conditions which allow to restrict the original problem posed on an unbounded domain to a bounded domain of interest. These bounds can be used to obtain the semi-classical limit of the system. The paper also gives an existence and uniqueness result for weak solutions while they were previously defined in a strong sense.
\end{abstract}

Key words. semiconductors, non-linear Schrdinger equation, open boundary conditions, uniform estimates

AMS subject classifications. 35Q40, 35Q55

\section{Introduction}

The modeling of semiconductors at the quantum level has become a very active area of research during the past decades. Indeed, the design of high-performance components requires the development of simulation tools that help engineers in finding the best configurations. This in turn demands a compromise between the accuracy of the models and the computational cost, and thus to derive models as close to the physics as possible with a relatively cheap cost of resolution. The particular geometry and physics of semiconductors allow for a wide variety of models, see for instance [8]. A semiconductor can roughly be decomposed into two zones: an access zone, through which the particles reach the active zone, where basically all the main physical effects take place. Whereas the access zone is generally not the most relevant part of the component, it has usually the largest dimensions (say some hundreds of nanometers long) and thus much computational time might be spent there. On the other hand, the active zone, which could be roughly a few tens of nanometers long, represents the essential part of the semiconductor and needs to be carefully treated. Indeed, the operation of the device is basically induced by the potential profile in the active region which presents some sharp variations - on the order of the De Broglie wavelength of the electrons - so that the dynamics requires a quantum description, more expensive than a kinetic one. It has then led to different strategies to lower the computational time spent in the access zones. One possible strategy is to prescribe adequate transparent boundary conditions at the interfaces access zone-active zone, so as to limit the resolution to the active zone; another strategy is to model the two zones differently - with a relatively cheap treatment of the access zone - and to couple them at the interfaces. The first strategy has already received great interest since it is related to many wave propagation problems in unbounded domains for which the aim is to restrict the resolution to a bounded zone of interest. Indeed, the Schrdinger equation governing the dynamics of the electrons can also be seen

\footnotetext{
*Received: April 17, 2007; accepted (in revised version); June 18, 2007. Communicated by Peter Markowich.

${ }^{\dagger}$ Universit de Lyon, Universit Lyon 1, CNRS, UMR 5208 Institut Camille Jordan/ISTIL, Btiment du Doyen Jean Braconnier, 43, blvd du 11 novembre 1918, F - 69622 Villeurbanne Cedex, France (pinaud@math.univ-lyon1.fr).
} 
as a paraxial approximation of the Helmholtz equation (see [30] for one of the first derivations) so that some existing results apply. There is very abundant literature about the subject: one could cite the pioneering work of [16], [3] for more recent advances, [4] in the context of semiconductors and [5] for numerical considerations, and for instance $[2,30]$ for applications to underwater acoustics. Note that the concept of open systems at the quantum level cannot be straightforwardly defined as it is at the kinetic level where it suffices to prescribe the distribution function for incoming velocities. This requires the introduction of conjuguate operators which dissociate ingoing from outgoing particles as was done elegantly in a very general framework in [24]. The second strategy is also an active area of research. Typically, the active region is treated as fully quantum, with possibly some subband decomposition, see [27], while the access zone only requires a kinetic description. The two descriptions are then connected via adequate interface conditions, as was done in $[14,9]$.

The description chosen in this paper is fully quantum, namely both in the access and active zones, and so falls into the first type of model. The dynamics of the electrons is then given by the Schrdinger equation everywhere in the semiconductor and it is assumed that their energy distribution is known. Since the electrons are charged particles, they self-interact. The non-linear effects are taken into account at the Hartree level through a potential solution to the Poisson equation, giving rise to the so-called Schrdinger-Poisson system. This system can be seen as a mean-field approximation of a system of many interacting particles through a Coulomb potential [7]. There is an extensive literature about the subject; see for instance [19] for a general mathematical analysis, and $[23,24]$ in the context of an open quantum system. In [12] a quantum transport model is introduced, and explicit boundary conditions at the interfaces access zone - active region are derived and will be recalled further in the paper. The wavefunctions are solution to the Schrdinger equation

$$
i \hbar \frac{\partial \psi_{\lambda}}{\partial t}=\mathcal{H}(t) \psi_{\lambda}
$$

where $\hbar$ is the Planck constant, $\lambda$ is a given quantum number and the Hamiltonian is defined by

$$
\mathcal{H}(t)=-\frac{\hbar^{2}}{2 m_{e}} \Delta+V_{e}(t, x)+V_{s}(t, x) .
$$

$m_{e}$ is the effective mass of the electron in the semiconductor (assumed constant for simplicity), $V_{e}$ is an exterior potential, while $V_{s}$ is the self-consistent potential solution to the Poisson equation

$$
-\Delta V_{s}=\int\left|\psi_{\lambda}\right|^{2} d \mu
$$

for some measure $\mu$. In [12], the model is shown to have a unique strong solution $\left(\psi_{\lambda}, V_{s}\right)$ (in the sense that the Schrdinger equation is verified almost everywhere in time and space) provided the data are regular enough. A possible way to confirm that the introduced transparent boundary conditions correctly describe the physics of the device is to take the semi-classical limit of the above system, by letting the scaled Planck constant $\varepsilon\left(\varepsilon:=\hbar:=\frac{\hbar}{\sqrt{m_{e}}}\right.$ in the sequel) go to zero. This limit is performed by means of Wigner transforms [32] which relate the quantum dynamics to the classical dynamics. It is thus expected that in the limit, the boundary conditions simply reduce 
to standard inflow boundary conditions, as was done in [11] for a one-dimensional stationary model. The Wigner transform is defined by, for any $\varphi \in L^{2}\left(\mathbb{R}^{d}\right)$,

$$
W^{\varepsilon}(x, k)=\frac{1}{(2 \pi)^{d}} \int_{\mathbb{R}^{d}} e^{i x \cdot k} \varphi\left(x-\frac{\varepsilon}{2} y\right) \varphi^{*}\left(x+\frac{\varepsilon}{2} y\right) d y,
$$

where $\varphi^{*}$ denotes the complex conjugate of $\varphi$ and $d$ the dimension. If $\varphi$ satisfies the time-dependent Schrdinger equation with potential $V(t, x)$, then its Wigner transform $W^{\varepsilon}$ is solution to the Wigner equation, namely

$$
\begin{aligned}
\frac{\partial W^{\varepsilon}}{\partial t}+k \cdot \nabla_{x} W^{\varepsilon}+K *_{k} W^{\varepsilon} & =0, \\
K(t, x, k) & =\frac{i}{(2 \pi)^{d}} \int_{\mathbb{R}^{d}} e^{-i k \cdot y} \varepsilon^{-1}\left(V\left(t, x+\frac{\varepsilon}{2} y\right)-V\left(t, x-\frac{\varepsilon}{2} y\right)\right) d y .
\end{aligned}
$$

Wigner transforms have found applications in many high-frequency asymptotic problems; see for instance [28] for a formal analysis of hyperbolic equations with random coefficients, $[6,17]$ for a semi-classical limit of random Schrdinger equations, [21, 22] for Schrdinger-Poisson systems and [13] for the Helmholtz equation. Passing formally to the limit in the above equation leads to the Vlasov equation,

$$
\frac{\partial W}{\partial t}+k \cdot \nabla_{x} W-\nabla_{x} V \cdot \nabla_{k} W=0
$$

where $W$ is the limit of $W^{\varepsilon}$ in some sense. The Vlasov equation then has to be supplemented at the interfaces with inflow boundary conditions of the type $W(t, x, k)=f(k)$ for entering wave vectors $k$, which will be the classical analogue of the quantum transparent boundary conditions. Passing rigorously to the limit requires some uniform in $\varepsilon$ bounds for the wave functions, which in turn provide estimates for the Wigner transform in some appropriate spaces, see $[18,21]$. The purpose of the present paper is then to address the question of uniform bounds for the open Schrdinger-Poisson system introduced in [12]. While in standard Schrdinger equations with $L^{2}$ initial conditions those estimates are straightforward, it is not the case when considering open systems with transparent boundary conditions. The reference [12] gives some regularity results and estimates, without specifying the dependence on $\varepsilon$. This work thus provides uniform bounds in $L_{\text {loc }}^{2}$, which stem from a careful analysis of the nonlocal in time boundary conditions imposed on the interfaces active zone - access zone. The semi-classical limit and the obtention of the inflow boundary conditions from the quantum transparent boundary conditions will be performed independently in a further work [26]. In addition to these estimates, we construct as well weak solutions to the open Schrdinger-Poisson model of [12], where the solutions were only defined in a strong sense. Those solutions verify the Schrdinger equation in a variational form which could be suitable for numerical simulations, since it naturally incorporates the transparent boundary conditions in the formulation.

The paper is organized as follows: in Section 2, we recall the transport model of [12]; in Section 3, we present the weak formulation and the main result, namely the uniform bounds; in Section 4, the proof of the theorem is given and finally one can find in Appendix A and B some technical results.

\section{Presentation of the problem}

We recall in this section the transport model introduced in [12] in a timedependent picture and in [10] in a stationary one. It consists of a Schrdinger-Poisson 
system posed on an unbounded domain, with non-vanishing conditions at infinity modeling the electron injection in the structure. This system is then reduced to a problem posed on a bounded domain with suitable inhomogeneous transparent boundary conditions taking into account the injected particles.

2.1. Geometry. The unbounded domain is denoted by $\Omega$ and its dimension by $d$. It is assumed in the sequel that $d=2$ or $d=3$. The domain $\Omega$ is then split into two zones, a bounded active zone denoted by $\Omega_{0}$ and an unbounded access zone, consisting of $n$ wave guides $\Omega_{j}, j=1, \ldots, n$, see Figure 2.1. The interfaces between $\Omega_{0}$ and each $\Omega_{j}$ are supposed to be flat and are denoted by $\Gamma_{j}$. The waveguides $\Omega_{j}$ have a cylinder-like structure and can thus be written as the cartesian product $\Gamma_{j} \mathbb{R}^{+}$. They are equipped with a local set of coordinates $\left(\xi_{j}, \eta_{j}\right) \in \Gamma_{j} \mathbb{R}^{+}$. Here, $\eta_{j}$ is basically the variable associated with the direction of propagation in the lead $j$. The outer boundaries of the $\Gamma_{j}$ 's are denoted by $\Gamma_{j, 0}$. The remaining part of the boundary of $\Omega_{0}$ is denoted by $\Gamma_{0}$ so that $\partial \Omega_{0}=\Gamma_{0} \bigcup\left(\cup_{j=1}^{n} \Gamma_{j}\right)$. We also introduce $\left(\mu_{j}\right)_{j=1, \ldots, n}$, a

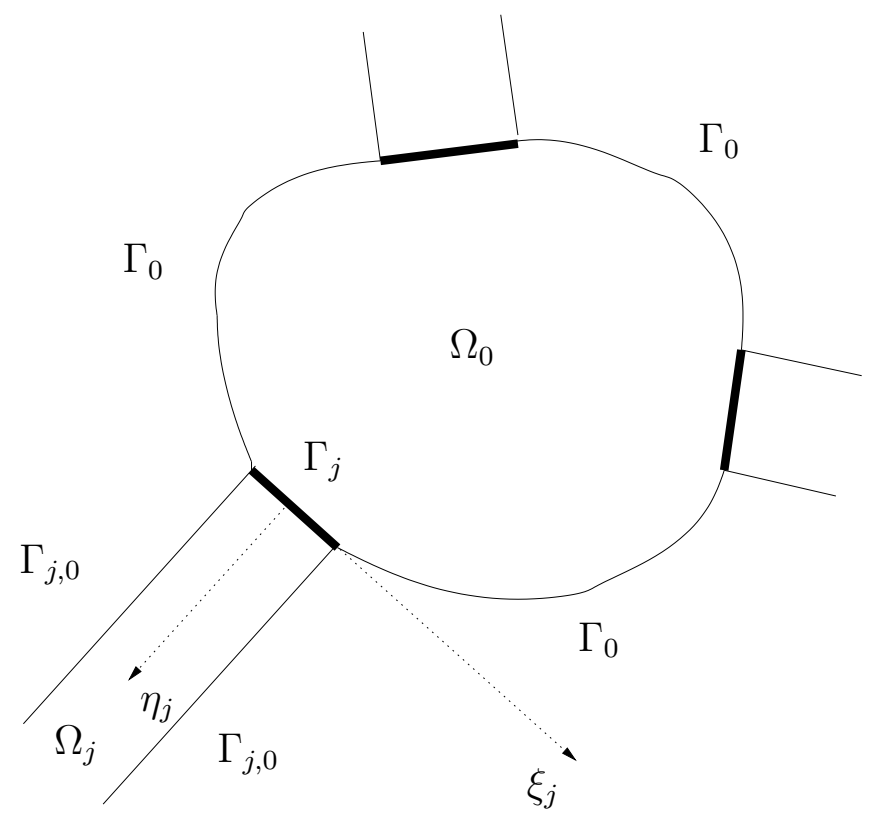

FIG. 2.1. The domain $\Omega$

partition of unity of $\Omega$, i.e. some $\mathcal{C}^{\infty}(\Omega)$ functions which satisfy

$$
\left\{\begin{array}{l}
0 \leq \mu_{j} \leq 1, \quad \sum_{j=1}^{n} \mu_{j}=1 \quad \text { on } \Omega \\
\mu_{j}=1 \text { on } \Omega_{j} \quad j=1, \ldots, n, \\
\mu_{j}=0 \text { on } \Omega_{k} \quad \text { for } k \neq 0, k \neq j .
\end{array}\right.
$$

2.2. Initial conditions. To model the electron injection, the initial conditions are supposed to be non-zero in the leads and to be scattering states of a given Hamiltonian $\mathcal{H}^{0}$ defined by

$$
\mathcal{H}^{0}=-\frac{\varepsilon^{2}}{2} \Delta+V^{0}
$$


where $V^{0}$ is an exterior potential which is assumed to depend only on the transversal coordinate in the leads $\Omega_{j}$, i.e.

$$
V^{0} \in L^{\infty}(\Omega) ;\left.\quad V^{0}\right|_{\Omega_{j}}=V^{0}\left(\xi_{j}\right) .
$$

The fact that $V^{0}$ does not depend on $\eta_{j}$ is necessary to be able to construct rather simple - though not obvious - boundary conditions on the interfaces $\Gamma_{j}, j \neq 0$. When $V^{0}$ is linear in $\eta_{j}$, the analysis is more involved and the resulting boundary conditions are more complex, see for instance [15]. Moreover, $V^{0}$ does not belong to any $L^{p}(\Omega)$, $p<\infty$, since it supposedly does not vanish when $\eta_{j} \rightarrow \infty$. We then define the transversal Hamiltonian $\mathcal{H}_{j}^{0}=-\frac{\varepsilon^{2}}{2} \Delta_{\xi_{j}}+V^{0}\left(\xi_{j}\right)$, equipped with Dirichlet boundary conditions on $\Gamma_{j, 0}$. It admits a compact resolvent and this leads to the following definition:

Definition 2.1. The transversal eigenmodes and the eigenvalues of the guide $j$ are defined by

$$
\left\{\begin{array}{l}
\mathcal{H}_{j}^{0} \chi_{m}^{0, j}=E_{m}^{j} \chi_{m}^{0, j}, \quad m \in \mathbb{N}^{*}, \quad j=1, \ldots, n, \\
\chi_{m}^{0, j} \in H_{0}^{1}\left(\Gamma_{j}\right), \quad \int_{\Gamma_{j}} \chi_{m}^{0, j} \overline{\chi_{m^{\prime}}^{0, j}} d \sigma_{j}=\delta_{m, m^{\prime}},
\end{array}\right.
$$

where $\sigma_{j}$ is the surface measure on $\Gamma_{j}$. Notice that we do not write explicitly the dependence of $\chi_{m}^{0, j}$ and $E_{m}^{j}$ on $\varepsilon$ for notational simplicity. For any fixed $j$ and $\varepsilon$, the sequence $\left(E_{m}^{j}\right)_{m}$ tends to $+\infty$ as $m$ tends to $+\infty$. For two functions $f$ and $g$ in $L^{2}\left(\Gamma_{j}\right)$, we define

$$
\langle f, g\rangle_{j}:=\int_{\Gamma_{j}} f\left(\xi_{j}\right) \bar{g}\left(\xi_{j}\right) d \sigma_{j}, \quad f_{m}^{j}:=\int_{\Gamma_{j}} f\left(\xi_{j}\right) \bar{\chi}_{m}^{j}\left(\xi_{j}\right) d \sigma_{j} .
$$

REMARK 2.1. Let $\varphi$ be an $L^{2}\left(\Gamma_{j}\right)$ function. The relation $\varphi \mapsto\left(\sum_{m \geq 1}\left(E_{m}^{j}\right)^{\alpha}\left|\varphi_{m}^{j}\right|^{2}\right)^{1 / 2}$ defines a norm equivalent to the $H^{\alpha}\left(\Gamma_{j}\right)$ norm.

We suppose without loss of generality that $E_{m}^{j} \geq 0, \forall m \geq 1$ and $\forall j \geq 1$. It suffices in the sequel to multiply the time-dependent Schrdinger equation by the phase factor $e^{i \frac{t}{\varepsilon} \min _{j, m} E_{m}^{j}}$ to recover the general case where $V_{j}$ is negative and bounded from below.

The electrons are injected in the leads in given quantum states. These states follow a prescribed statistics denoted by $\mu . \mu$ is a non-negative measure on the state space $\Lambda$, and a pure state is denoted by $\lambda$. The wave functions are thus indexed by $\lambda$. Consider the following hypotheses for a family of functions $\psi_{\lambda}^{0} \in H_{\mathrm{loc}}^{1}(\Omega)$ :

(H-1) For a.e. $\lambda \in \Lambda$, there exists a constant $E(\lambda)$ such that

$$
\mathcal{H}^{0} \psi_{\lambda}^{0}=E(\lambda) \psi_{\lambda}^{0} \quad \text { on } \Omega_{j}, \quad \psi_{\lambda}^{0}=0 \quad \text { on } \Gamma_{j, 0} \mathbb{R}_{+}, \quad j \neq 0 .
$$

$ß(\mathbf{H}-2)$ For any bounded set $K \subset \Omega$, there exists $C_{K}>0$ finite such that

$$
\int_{\Lambda}\left\|\psi_{\lambda}^{0}\right\|_{H^{1}(K)}^{2} d \mu(\lambda) \leq C_{K} .
$$

In practice, the electrons are injected in the guide $j_{0}$, on the transverval mode $m_{0}$ and with a non-vanishing longitudinal momentum $p$. This implies that $\lambda=\left\{p, m_{0}, j_{0}\right\}$, $\Lambda=\mathbb{R}_{+}^{*} \mathbb{N}^{*}\{1, \ldots, n\}, E(\lambda)=\frac{1}{2} p^{2}+E_{m_{0}}^{j_{0}}$ and

$$
d \mu(\lambda)=\Phi\left(p, m_{0}, j_{0}\right) d p \delta\left(m_{0}\right) \delta\left(j_{0}\right),
$$


where $\delta$ denotes the Dirac measure and the positive function $\Phi \in L^{1}\left(\mathbb{R}_{+}^{*}, \ell^{1}\left(\mathbb{N}^{*}\{1, \ldots, n\}\right)\right)$ denotes the statistics of the injected electrons, typically Fermi-Dirac statistics. This is equivalent to writing, for any $\varphi \in L^{1}(\Lambda, d \mu)$,

$$
\int_{\Lambda} \varphi(\lambda) d \mu(\lambda)=\sum_{j_{0}=1}^{n} \sum_{m_{0}=1}^{\infty} \int_{\mathbb{R}^{+}} \varphi\left(p, m_{0}, j_{0}\right) \Phi\left(p, m_{0}, j_{0}\right) d p .
$$

The energy $\frac{1}{2} p^{2}$ represents the longitudinal kinetic energy of the electrons, while $E_{m_{0}}^{j_{0}}$ is the transversal energy in the lead $j_{0}$. We add the following hypotheses on the measure $\mu$,

$$
\int_{\Lambda}\left(1+p^{5}\right) d \mu<+\infty
$$

and a local in $\lambda$ version of $(\mathbf{H}-\mathbf{2})$ :

(H-3) for any bounded set $K \subset \Omega$, there exists $C_{K}^{\prime}>0$ finite and independent of $\lambda$ such that

$$
\Phi(\lambda)\left\|\psi_{\lambda}^{0}\right\|_{H^{1}(K)}^{2} \leq C_{K}^{\prime} .
$$

A family $\psi_{\lambda}^{0} \in H_{\text {loc }}^{1}(\Omega)$ indexed by $\lambda \in \Lambda$ is then said to belong to the class of initial data if hypothesis $(\mathbf{H}-\mathbf{1})-(\mathbf{H}-\mathbf{3})$ are satisfied.

Transparent boundary conditions for the initial conditions. It is proved in [10], that wave functions satisfying hypothesis (H-1) verify some boundary conditions on $\Gamma_{j}$, allowing for a simplification to a boundary value problem on the bounded domain $\Omega_{0}$. Analogous boundary conditions have been obtained for the one-dimensional case in [11]. The explicit form of these stationary boundary conditions is needed in the sequel to carefully analyze their time-dependent version. We thus briefly describe their form and derivation now; the details can be found in [10].

The restriction of $\psi_{\lambda}^{0}$ to $\Omega_{j}$ is projected on the transversal basis $\left(\chi_{m}^{0, j}\right)_{m}$, i.e. $\left.\psi_{\lambda}^{0}\right|_{\Omega_{j}}\left(\xi_{j}, \eta_{j}\right)=\sum_{m} \chi_{m}^{0, j}\left(\chi_{j}\right) f^{j}\left(\eta_{j}\right)$, so that $f^{j}$ verifies, according to $(\mathbf{H}-\mathbf{1})$,

$$
-\frac{\varepsilon^{2}}{2} \frac{\partial^{2} f^{j}}{\partial \eta_{j}^{2}}=\left(E(\lambda)-E_{m}^{j}\right) f^{j}, \quad \eta_{j} \in \mathbb{R}^{+}
$$

and thus reads

$$
f^{j}\left(\eta_{j}\right)=a_{m}^{j} \exp \left(-\frac{i \eta_{j}}{\varepsilon} \sqrt[+]{2\left(E(\lambda)-E_{m}^{j}\right)}\right)+b_{m}^{j} \exp \left(\frac{i \eta_{j}}{\varepsilon} \sqrt[+]{2\left(E(\lambda)-E_{m}^{j}\right)}\right) .
$$

Above, $\sqrt[+]{ }$ denotes the complex square root with a non-negative imaginary part, $b_{m}^{j}$ is an unknown coefficient depending on the solution and $a_{m}^{j}$ is the amplitude of the injected electrons travelling towards the active region and is thus known. We suppose the electrons are injected in the lead $j_{0}$, with a momemtum $p$, on the transversal mode $m_{0}$ and with an amplitude one, so that $a_{m}^{j}=\delta_{j}^{j_{0}} \delta_{m}^{m_{0}}$ and $E(\lambda)=\frac{1}{2} p^{2}+E_{m_{0}}^{j_{0}}$. The modes associated with $E_{m}^{j}<E(\lambda)$ are the propagating modes and the modes with $E_{m}^{j}>E(\lambda)$ are the evanescent modes. When $j=j_{0}, b_{m}^{j}$ is a reflection coefficient, and when $j \neq j_{0}$, it is a transmission coefficient. The boundary conditions are obtained by eliminating $b_{m}^{j}$ and formally read

$$
\left.\varepsilon \frac{\partial \psi_{\lambda}^{0}}{\partial \eta_{j}}\right|_{\Gamma_{j}}=\mathbb{Z}_{j}[E(\lambda)]\left(\psi_{\lambda}^{0}\right)+\mathbb{S}_{j}[E(\lambda)]
$$


These relations are impedance-like boundary conditions. The operator $\mathbb{Z}_{j}[E(\lambda)]$ corresponds to an homogeneous part while $\mathbb{S}_{j}[E(\lambda)]$ is a source term. They read

$$
\begin{aligned}
\mathbb{Z}_{j}[E(\lambda)]\left(\psi_{\lambda}^{0}\right) & =i \sum_{m=1}^{\infty} \sqrt[+]{2\left(E(\lambda)-E_{m}^{j}\right)} \psi_{m}^{0, j} \chi_{m}^{0, j}\left(\xi_{j}\right), \\
\mathbb{S}_{j}[E(\lambda)] & =-2 i \delta_{j}^{j_{0}} p \chi_{m_{0}}^{0, j}\left(\xi_{j}\right), \\
\psi_{m}^{0, j} & =\left\langle\psi_{\lambda}^{0}\left(\eta_{j}=0, \cdot\right), \chi_{m}^{0, j}\right\rangle_{j} .
\end{aligned}
$$

It is shown in [10] that these boundary conditions actually make sense in a weak formulation for every $\psi_{\lambda}^{0} \in H^{1}\left(\Omega_{0}\right)$; see therein for more details and a complete analysis of the related stationary open Schrdinger-Poisson system.

2.3. Potentials. In [12], it is assumed that the exterior potential of the Hamiltonian (1.1) shares properties close to that of $V^{0}$. In order to solve exactly the Schrdinger equation in the leads for the derivation of the boundary conditions, it is supposed that the spatial dependence of the exterior potential $V_{e}$ is only transversal. The following class is then introduced in [12]: a given potential $V$ belongs to the class $\mathcal{V}$ if it satisfies:

1. $V \in \mathcal{C}^{1}\left([0, T], L^{\infty}(\Omega)\right)$,

2. for any $j=1, \ldots, n$, there exists a function $V_{j}(t)$ such that for $x \in \Omega_{j}$ we have $V(t, x)=V^{0}(x)+V_{j}(t)$.

The regularity in time is needed to obtain energy estimates, that is, $H^{1}\left(\Omega_{0}\right)$ bounds for the wavefunction. One could also consider non-regular in time potentials with some Sobolev regularity in space, but in the typical application we are interested innamely quantum transport in nanostructures - the potentials present a barrier profile which is obviously not smooth.

\section{The transient open Schrdinger-Poisson system}

This consists in solving for $V_{s}(t, x)$ and $\psi_{\lambda}(t, x)$ the following system

$$
\begin{aligned}
i \varepsilon \partial_{t} \psi_{\lambda} & =\mathcal{H}(t) \psi_{\lambda} ; \quad \psi_{\lambda}(0, \cdot)=\psi_{\lambda}^{0} ; \quad x \in \Omega ; \quad \lambda \in \Lambda, \\
\mathcal{H}(t) & =-\frac{\varepsilon^{2}}{2} \Delta+V_{e}(t, x)+V_{s}(t, x), \\
\psi_{\lambda} & =0 ; \quad x \in \Gamma_{0} \bigcup\left(\cup_{j=1}^{n}\left(\Gamma_{j, 0} \mathbb{R}^{+}\right)\right), \\
-\Delta V_{s} & =\int_{\Lambda}\left|\psi_{\lambda}\right|^{2} d \mu(\lambda), \quad x \in \Omega_{0} ;\left.\quad V_{s}\right|_{\partial \Omega_{0}}=0,
\end{aligned}
$$

where $V_{e}$ belongs to the class of potentials $\mathcal{V}$ and $\psi_{\lambda}^{0}$ belongs to the class of initial conditions. Notice that the Schrdinger equation is set on the whole domain $\Omega$ including the leads. As was done for the stationary case in [10] and in [11], it is proven in [12], thanks to the introduction of suitable boundary conditions, that this system is equivalent to the same Schrdinger-Poisson system posed only in the domain $\Omega_{0}$. In the next subsection, we recall the time-dependent transparent boundary conditions introduced in [12] and the theorem stating the existence and uniqueness of strong solutions to (3.1)-(3.4) in the sense of [25]. We then present the weak formulation of (3.1)-(3.4) and state the main result of the paper. 
3.1. Transparent boundary conditions and strong solutions. We first introduce some notations. Let

$$
\chi_{m}^{j}\left(t, \xi_{j}\right):=\chi_{m}^{0, j}\left(\xi_{j}\right) \exp \left(-\frac{i}{\varepsilon} \int_{0}^{t}\left(V_{j}(\tau)+E_{m}^{j}\right) d \tau\right) .
$$

At any time, $\left(\chi_{m}^{j}\right)_{m \geq 1}(t,$.$) is an orthonormal basis of L^{2}\left(\Gamma_{j}\right)$.

Definition 3.1. For any given function $f \in H^{1 / 2}(0, T)$, one defines-see [20], or [29]:

$$
\partial^{1 / 2} f:=\frac{1}{\sqrt{\pi}} \frac{d}{d t} \int_{0}^{t} \frac{f(\tau)}{\sqrt{t-\tau}} d \tau=\frac{1}{\sqrt{\pi}} \frac{d}{d t} \mathcal{I}^{1 / 2} f .
$$

$H^{1 / 2}(0, T)$, see [1], is the fractional Sobolev space of functions

$$
H^{1 / 2}(0, T)=\left\{f \in L^{2}(0, T) \quad \text { such that } \int_{0}^{T} \int_{0}^{T} \frac{|f(s)-f(\tau)|^{2}}{(s-\tau)^{2}} d s d \tau<\infty\right\} .
$$

An alternative definition that will be used in the sequel defines $H^{1 / 2}(0, T)$ as the restriction to $(0, T)$ of functions belonging to $H^{1 / 2}(\mathbb{R})$. Then, for any $f \in$ $H^{1 / 2}\left((0, T), L^{2}\left(\Gamma_{j}\right)\right)$, we set

$$
\mathbb{D}_{j}^{1 / 2} f\left(t, \xi_{j}\right):=\sqrt{2} \sum_{m \geq 1} \chi_{m}^{j}\left(t, \xi_{j}\right) \partial^{1 / 2}\left\langle f(t, \cdot), \chi_{m}^{j}(t, \cdot)\right\rangle_{j} .
$$

Let now

$$
\begin{aligned}
\psi_{\lambda}^{p w} & :=\psi_{\lambda}^{0} \sum_{j=1}^{n} \mu_{j} \theta_{\lambda}^{j}, \\
\theta_{\lambda}^{j}(t) & :=\exp \left(-\frac{i}{\varepsilon} \int_{0}^{t}\left(E(\lambda)+V_{j}(s)\right) d s\right),
\end{aligned}
$$

where $\left(\mu_{j}\right)_{j}$ is the partition of unity introduced in Section 2.1. Then, according to [12], the wave function $\psi_{\lambda}$ satisfies the following boundary conditions:

$$
\frac{\partial}{\partial \eta_{j}}\left(\psi_{\lambda}-\psi_{\lambda}^{p w}\right)=-\frac{e^{-i \pi / 4}}{\sqrt{\varepsilon}} \mathbb{D}_{j}^{1 / 2}\left(\psi_{\lambda}-\psi_{\lambda}^{p w}\right) ; \quad x \in \Gamma_{j}, \quad j=1, \ldots, n .
$$

One can also write a boundary condition involving a half-integral $\mathcal{I}^{1 / 2}$ rather than a half-derivative. The final system we will deal with in the sequel thus couples many Schrdinger equations, posed on a bounded domain with open boundary conditions, to the Poisson equation. The complete system reads:

$$
\begin{aligned}
i \varepsilon \partial_{t} \psi_{\lambda} & =\mathcal{H}(t) \psi_{\lambda} ; \quad \psi_{\lambda}(0, \cdot)=\psi_{\lambda}^{0} ; \quad x \in \Omega_{0} ; \quad \lambda \in \Lambda, \\
\frac{\partial}{\partial \eta_{j}}\left(\psi_{\lambda}-\psi_{\lambda}^{p w}\right) & =-\frac{e^{-i \pi / 4}}{\sqrt{\varepsilon}} \mathbb{D}_{j}^{1 / 2}\left(\psi_{\lambda}-\psi_{\lambda}^{p w}\right) ; \quad x \in \Gamma_{j}, \\
\psi_{\lambda} & =0 ; \quad x \in \Gamma_{0}, \\
-\Delta V_{s} & =\int_{\Lambda}\left|\psi_{\lambda}\right|^{2} d \mu(\lambda) ; \quad x \in \Omega_{0} ;\left.\quad V_{s}\right|_{\partial \Omega_{0}}=0 .
\end{aligned}
$$


The existence result of [12] provides strong solutions to (3.8)-(3.11) in the sense that the Schrdinger equation and the boundary conditions are satisfied almost everywhere on $\Omega_{0}$ and on $\Gamma_{j}$, respectively. The exact statement is the following:

TheOREM 3.1. [12]. Let $V_{e} \in \mathcal{V}, \psi_{\lambda}^{0}$ verifying hypothesis $(\mathbf{H}-\mathbf{1}), d=2$ or $d=3$, and in addition

$$
\int_{\Lambda}\left\|\psi_{\lambda}^{0}\right\|_{H^{2}(K)}^{2} d \mu(\lambda)<\infty ; \quad \Phi \text { has a compact support, }
$$

for any bounded set $K \subset \Omega$. Then (3.8)-(3.11) is equivalent to (3.1)-(3.4) and there exists a unique solution $\left(\psi_{\lambda}, V_{s}\right)$ to (3.1)-(3.4) such that

$$
\begin{aligned}
& \psi_{\lambda} \in \psi_{\lambda}^{p w}+\mathcal{C}^{0}\left([0, T], H^{2}(\Omega)\right) \cap \mathcal{C}^{1}\left([0, T], L^{2}(\Omega)\right), \quad \lambda a . e . \\
& V_{s} \in \mathcal{C}^{0}\left([0, T], H_{0}^{1}(\Omega)\right) \cap \mathcal{C}^{0}\left([0, T], H^{4}(\Omega)\right) \cap \mathcal{C}^{1}\left([0, T], H^{2}(\Omega)\right),
\end{aligned}
$$

for any positive arbitrary large $T$ and so that (3.8) and (3.9) are verified almost everywhere.

The above theorem does not provide any information about the dependence on $\varepsilon$ of the different bounds on $\psi_{\lambda}$ and $V_{s}$, which is paramount for the semi-classical limit. The proof can actually be adapted to yield more regularity of the solution when the data are more regular; for instance we can get pointwise in $\lambda, \psi_{\lambda} \in \mathcal{C}^{\infty}([0, T] \Omega)$ when $\psi_{\lambda}^{0} \in \mathcal{C}^{\infty}(\Omega)$ and $V_{e} \in \mathcal{C}^{\infty}([0, T] \Omega)$. We will use this regularity further to use $\psi_{\lambda}$ as a test function in the weak formulation. Some details about the regularization procedure are given in Appendix B.

3.2. Weak formulation and main result. We present in this section the weak formulation of (3.8)-(3.11) and the main result of the paper. The formulation of the boundary terms requires particular attention to obtain the $\varepsilon$-independent estimates. More precisely, the boundary condition (3.9) is split into homogeneous and inhomogeneous parts, that is,

$$
\frac{\partial \psi_{\lambda}}{\partial \eta_{j}}=-\frac{e^{-i \pi / 4}}{\sqrt{\varepsilon}} \mathbb{D}_{j}^{1 / 2}\left(\psi_{\lambda}\right)+{ }_{j}^{\varepsilon}\left(\psi_{\lambda}^{p w}\right),
$$

where

$$
\begin{aligned}
{ }_{j}^{\varepsilon}\left(\psi_{\lambda}^{p w}\right) & :=\left.\frac{\partial \psi_{\lambda}^{p w}}{\partial \eta_{j}}\right|_{\Gamma_{j}}+\frac{e^{-i \pi / 4}}{\sqrt{\varepsilon}} \mathbb{D}_{j}^{1 / 2}\left(\psi_{\lambda}^{p w}\right) \\
& =\frac{1}{\varepsilon}\left(\mathbb{Z}_{j}[E(\lambda)]\left(\psi_{\lambda}^{0}\right)+\mathbb{S}_{j}[E(\lambda)]\right) \theta_{\lambda}^{j}+\frac{e^{-i \pi / 4}}{\sqrt{\varepsilon}} \mathbb{D}_{j}^{1 / 2}\left(\psi_{\lambda}^{p w}\right) .
\end{aligned}
$$

Note here that we used the stationary open boundary conditions (2.3) to define ${ }_{j}^{\varepsilon}\left(\psi_{\lambda}^{p w}\right)$. The solutions to (3.8) are sought under the following weak form: let $u \in \mathcal{C}^{1}\left([0, T), H^{1}\left(\Omega_{0}\right)\right)$ be a test function, where $T$ is an arbitrary non-negative constant; denoting by $(\cdot, \cdot)$ the $L^{2}\left(\Omega_{0}\right)$ inner product and using the Green formula and the boundary conditions (3.9) and (3.10), we find $\lambda$ a.e.,

$$
-i \varepsilon \int_{0}^{T}\left(\psi_{\lambda}, \partial_{s} u\right) d s=i \varepsilon\left(\psi_{\lambda}^{0}, u(0, \cdot)\right)+\frac{1}{2} \varepsilon^{2} \int_{0}^{T}\left(\nabla \psi_{\lambda}, \nabla u\right) d s+\int_{0}^{T}\left(V \psi_{\lambda}, u\right) d s
$$




$$
+\frac{1}{2} \sum_{j=1}^{n}\left[\varepsilon^{3 / 2} e^{-i \pi / 4} \int_{0}^{T}\left\langle\mathbb{D}_{j}^{1 / 2}\left(\psi_{\lambda}\right), u\right\rangle_{j} d s-\varepsilon^{2} \int_{0}^{T}\left\langle{ }_{j}^{\varepsilon}\left(\psi_{\lambda}^{p w}\right), u\right\rangle_{j} d s\right] .
$$

When the potential $V_{e}$ belongs to the class $\mathcal{V}$, it is rather natural to consider wavefunctions $\psi_{\lambda}$ solving (3.13) belonging to $L^{2}\left((0, T), H^{1}\left(\Omega_{0}\right)\right) \cap \mathcal{C}^{0}\left([0, T], L^{2}\left(\Omega_{0}\right)\right)$. Nevertheless, this regularity is not sufficient since the boundary terms need more integrability in time to make sense. To define the convenient functional space, we introduce the following family of unitary transformations: for any $f \in L^{2}\left((0, T), L^{2}\left(\Gamma_{j}\right)\right)$, let

$$
\mathcal{T}_{j} f\left(t, \xi_{j}\right):=\sum_{m \geq 1} e^{\frac{i}{\varepsilon} \int_{0}^{t}\left(E_{m}^{j}+V_{j}(s)\right) d s}\left\langle f(t, \cdot), \chi_{m}^{0, j}\right\rangle_{j} \chi_{m}^{0, j}\left(\xi_{j}\right)
$$

and let $\left(\mathcal{T}_{j} f\right)_{m}:=\left\langle\mathcal{T}_{j} f, \chi_{m}^{0, j}\right\rangle$. Consider now the functional space

$$
\begin{aligned}
\mathrm{E}=\left\{\varphi \in L^{2}\left((0, T), H^{1}\left(\Omega_{0}\right)\right)\right. & \cap \mathcal{C}^{0}\left([0, T], L^{2}\left(\Omega_{0}\right)\right), \quad \text { such that } \\
& \left.\mathcal{T}_{j} \varphi \in H^{1 / 4}\left((0, T), L^{2}\left(\Gamma_{j}\right)\right), \quad j=1, \ldots, n\right\},
\end{aligned}
$$

and let $\mathrm{E}^{0}$ be the space of functions belonging to $\mathrm{E}$ with a vanishing trace on $\Gamma_{0}$, i.e.

$$
\mathrm{E}^{0}=\left\{\varphi \in \mathrm{E}, \text { such that } \varphi_{\mid \Gamma_{0}}=0\right\} .
$$

In the weak formulation (3.13), the boundary term $\int_{0}^{T}\left\langle\mathbb{D}_{j}^{1 / 2}\left(\psi_{\lambda}\right), u\right\rangle d s$ has to be understood in the following weak sense, which uses the expression of the half-derivative in the Fourier space given in Lemma A.1 of Appendix A:

$$
\int_{0}^{T}\left\langle\mathbb{D}_{j}^{1 / 2}\left(\psi_{\lambda}\right), u\right\rangle d s=\frac{e^{i \pi / 4}}{2 \pi} \sum_{m \geq 1} \int_{\mathbb{R}}-\sqrt{\xi} \mathcal{F}\left(\widetilde{\left.\mathcal{T}_{j} \psi_{\lambda}\right)_{m}} \overline{\mathcal{F}\left(\widetilde{\left.\mathcal{T}_{j} u\right)_{m}}\right.} d \xi .\right.
$$

Above, the sign is the extension by 0 outside $[0, T], \mathcal{F}$ stands for the Fourier transform with respect to time and $\bar{\gamma}$ is the complex square root with non-positive imaginary part. The dual variable of $t$ is denoted by $\xi$. This expression is well-defined for any $\psi_{\lambda} \in \mathrm{E}$ and $u \in \mathcal{C}^{1}\left([0, T], H^{1}\left(\Omega_{0}\right)\right)$.

We state now the main result of the paper, which provides existence and uniqueness for the system (3.11)-(3.13) as well as uniform bounds in $\varepsilon$ for the density $n$ and energy $\mathcal{E}$ defined below:

TheOrem 3.2. Let $\psi_{\lambda}^{0}$ belongs to the class of initial data, let $V_{e} \in \mathcal{V}$ and assume $\mu$ satisfies (2.2). Let

$$
\begin{aligned}
n(t) & :=\int_{\Lambda}\left\|\psi_{\lambda}(t, \cdot)\right\|_{L^{2}\left(\Omega_{0}\right)}^{2} d \mu \\
\mathcal{E}(t) & :=\frac{\varepsilon^{2}}{2} \int_{\Lambda}\left\|\nabla \psi_{\lambda}(t, \cdot)\right\|_{L^{2}\left(\Omega_{0}\right)}^{2} d \mu+\frac{1}{2}\left\|\nabla V_{s}(t, \cdot)\right\|_{L^{2}\left(\Omega_{0}\right)}^{2} .
\end{aligned}
$$

Then the Schrdinger-Poisson system (3.11)-(3.13) admits a unique solution, for $d=2$ or $d=3$, such that, $\lambda$ a.e.,

$$
V_{s} \in L^{2}\left((0, T), W^{3, r}\left(\Omega_{0}\right)\right) \cap H^{1}\left((0, T), W^{1, r}\left(\Omega_{0}\right)\right) ; \quad \psi_{\lambda} \in E^{0},
$$


with $r<2$ when $d=2$ and $r=\frac{3}{2}$ when $d=3$. Moreover, assuming that

$$
n(0)+\mathcal{E}(0)+\varepsilon\left|\sum_{j=1}^{n} \int_{\Lambda}\left\langle\mathbb{Z}_{j}[E(\lambda)]\left(\psi_{\lambda}^{0}\right), \psi_{\lambda}^{0}\right\rangle_{j} d \mu\right| \leq C_{0},
$$

where $C_{0}$ is independent of $\varepsilon$, there exists $C_{1}$, depending on $C_{0}$, on $\left\|V_{e}\right\|_{\mathcal{C}^{1}\left([0, T], L^{\infty}(\Omega)\right)}$, on $\|p\|_{L^{5}(\Lambda, d \mu)}$, on $r$, and independent of $\varepsilon$ also, such that

$$
\|n\|_{L^{\infty}(0, T)}+\|\mathcal{E}\|_{L^{1}(0, T)}+\left\|\partial_{t} V_{s}\right\|_{L^{2}\left((0, T), L^{r}\left(\Omega_{0}\right)\right)} \leq C_{1},
$$

where $r<2$ when $d=2$ and $r<\frac{3}{2}$ when $d=3$.

The proof of the theorem is the object of the next section. The existence and uniqueness part is very standard and is obtained after regularization of the problem in order to use Theorem 3.1. Some estimates then give some compactness results and allow to pass to the limit in the weak formulation. The proof of the $\varepsilon$ independent estimates is more involved and requires a careful analysis of the boundary term ${ }_{j}^{\varepsilon}\left(\psi_{\lambda}^{p w}\right)$. Indeed, the term $\varepsilon\left\langle_{j}^{\varepsilon}\left(\psi_{\lambda}^{p w}\right), \psi_{\lambda}\right\rangle_{j}$ can be straightforwardly bounded by $C_{0}\left\|\psi_{\lambda}\right\|_{L^{2}\left(\Gamma_{j}\right)}\left\|\psi_{\lambda}^{0}\right\|_{H^{1 / 2}\left(\Gamma_{j}\right)}$ for some positive constant $C_{0}$ independent of $\varepsilon$; by means of trace theorems, this bound turns into $\varepsilon^{-1 / 2} C_{1}\left\|\psi_{\lambda}\right\|_{L^{2}\left(\Omega_{0}\right)}^{1 / 2}\left\|\varepsilon \nabla \psi_{\lambda}\right\|_{H^{1}\left(\Omega_{0}\right)}^{1 / 2}\left\|\psi_{\lambda}^{0}\right\|_{H^{1 / 2}\left(\Gamma_{j}\right)}$, which has a wrong homogeneity in $\varepsilon$ whatever the available bound on $\psi_{\lambda}^{0}$. We thus expect some compensation or averaging between the homogeneous stationary boundary conditions given by $\mathbb{Z}_{j}$ and the homogeneous time-dependent boundary conditions involving the half-derivative, which will allow control of the boundary terms in terms of other boundary terms with a sign argument. That property is shown in the next proposition. The $\varepsilon$-independent estimates rely as well on the verification of assumption (3.16). If the initial condition $\psi_{\lambda}^{0}$ is a solution to the Schrdinger equation not only in the leads but also in $\Omega_{0}$, as it is in [10], then (3.16) has to be verified by the solution to a stationary open Schrdinger equation. This property has only been shown until now in the one-dimensional case, in [11].

We decided in the theorem to set the transparent boundary conditions on the $\Gamma_{j}$ 's, while they could have been set anywhere further in the guides. Doing so would have led to $L_{\text {loc }}^{2}(\Omega)$ estimates for the density and for the energy.

Proposition 3.2.1. Let ${ }_{j}^{\varepsilon}$ be defined as in (3.12), $\psi_{\lambda}^{\text {pw }}$ as in $(3.6), \mathbb{S}_{j}[E(\lambda)]$ as in (2.5) and $\theta_{\lambda}^{j}(t)$ as in (3.7). Then, for any $t>0$, for $\psi_{\lambda}^{0} \in H^{1 / 2}\left(\Gamma_{j}\right), u \in L^{2+\delta}\left((0, t), L^{2}\left(\Gamma_{j}\right)\right)$, $\delta>0$, we have, $\lambda$ a.e,

$$
\int_{0}^{t}\left\langle{ }_{j}^{\varepsilon}\left(\psi_{\lambda}^{p w}\right), u\right\rangle_{j} d s=\frac{1}{\varepsilon} \int_{0}^{t}\left\langle\mathbb{S}_{j}[E(\lambda)] \theta_{\lambda}^{j}, u\right\rangle_{j} d s
$$

Proof. Setting

$$
\mathbb{B}_{j}\left(\psi_{\lambda}^{p w}\right)=\frac{1}{\varepsilon} \mathbb{Z}_{j}[E(\lambda)]\left(\psi_{\lambda}^{0}\right) \theta_{\lambda}^{j} ; \quad \mathbb{C}_{j}\left(\psi_{\lambda}^{p w}\right)=\frac{e^{-i \pi / 4}}{\sqrt{\varepsilon}} \mathbb{D}_{j}^{1 / 2}\left(\psi_{\lambda}^{p w}\right),
$$

(3.18) is equivalent to showing that $\int_{0}^{t}\left\langle\mathbb{B}_{j}\left(\psi_{\lambda}^{p w}\right)+\mathbb{C}_{j}\left(\psi_{\lambda}^{p w}\right), u\right\rangle_{j} d s=0$. Let $u_{m}^{j}(t)$ be the projection $u_{m}^{j}(t)=\left\langle u(t, \cdot), \chi_{m}^{0, j}\right\rangle_{j}$. Then, plugging the definition of $\mathbb{Z}_{j}(2.4)$ into 
$\mathbb{B}_{j}$ and denoting $\gamma_{m}^{j}=2\left(E(\lambda)-E_{m}^{j}\right)$, it follows that

$$
\begin{aligned}
\int_{0}^{t}\left\langle\mathbb{B}_{j}\left(\psi_{\lambda}^{p w}\right), u\right\rangle_{j} d s & =\frac{i}{\varepsilon} \sum_{m} \sqrt[+]{\gamma_{m}^{j}} \psi_{m}^{0, j} \int_{0}^{t} e^{-\frac{i}{\varepsilon} \int_{0}^{s}\left(E(\lambda)+V_{j}(\tau)\right) d \tau} \overline{u_{m}^{j}}(s) d s, \\
& =\frac{i}{\varepsilon} \sum_{m} \sqrt[+]{\gamma_{m}^{j}} \psi_{m}^{0, j} \overline{\mathcal{F} U_{m}^{j}}\left(-\gamma_{m}^{j} /(2 \varepsilon)\right) .
\end{aligned}
$$

Above, $\mathcal{F}$ denotes the Fourier transform with respect to time and $U_{m}^{j}$ is defined as

$$
U_{m}^{j}(s)=\left\{\begin{array}{l}
u_{m}^{j}(s) e^{\frac{i}{\varepsilon} \int_{0}^{s}\left(E_{m}^{j}+V_{j}(\tau)\right) d \tau} \quad \text { if } \quad s \in[0, t], \\
0 \quad \text { if } \quad s \notin[0, t] .
\end{array}\right.
$$

Concerning $\mathbb{C}_{j}$, invoking (3.5) yields

$$
\begin{aligned}
\int_{0}^{t}\left\langle\mathbb{C}_{j}\left(\psi_{\lambda}^{p w}\right), u\right\rangle_{j} d s & =\frac{\sqrt{2} e^{-i \pi / 4}}{\sqrt{\varepsilon}} \sum_{m} \psi_{m}^{0, j} \int_{0}^{t} e^{-\frac{i}{\varepsilon} \int_{0}^{s}\left(E_{m}^{j}+V_{j}(\tau)\right) d \tau} \overline{u_{m}^{j}}(s) \partial^{\frac{1}{2}}\left(e^{-\frac{i s}{2 \varepsilon} \gamma_{m}^{j}}\right) d s \\
& =\frac{\sqrt{2}}{2 \pi \sqrt{\varepsilon}} \sum_{m} \psi_{m}^{0, j} \int_{\mathbb{R}} \overline{\mathcal{F} U_{m}^{j}}(\xi) \sqrt{\xi} \mathcal{F}\left[\mathbb{1}_{\mathbb{R}^{+}} e^{-\frac{i}{2 \varepsilon} \gamma_{m}^{j} s}\right](\xi) d \xi,
\end{aligned}
$$

where we used the Fourier-Plancherel equality and (A.1). Above, $\bar{\gamma}$ is the complex square root with non-positive imaginary part. Actually, in the distribution sense,

$$
\mathcal{F}\left[\mathbb{1}_{\mathbb{R}^{+}} e^{-\frac{i}{2 \varepsilon} \gamma_{m}^{j} s}\right](\xi)=\pi \delta\left(\gamma_{m}^{j} /(2 \varepsilon)+\xi\right)-\text { p.v. } \frac{i}{\gamma_{m}^{j} /(2 \varepsilon)+\xi},
$$

where p.v. stands for the principal value. Then,

$$
\begin{aligned}
\int_{0}^{t}\left\langle\mathbb{C}_{j}\left(\psi_{\lambda}^{p w}\right), u\right\rangle_{j} d s= & \frac{1}{2 \varepsilon} \sum_{m} \sqrt{-\gamma_{m}^{j}} \psi_{m}^{0, j} \overline{\mathcal{F} U_{m}^{j}}\left(-\gamma_{m}^{j} /(2 \varepsilon)\right) \\
& -\frac{i \sqrt{2}}{2 \pi \sqrt{\varepsilon}} \sum_{m} \psi_{m}^{0, j} \text { p.v. } \int_{\mathbb{R}} \overline{\mathcal{F} U_{m}^{j}}(\xi) \frac{\sqrt{\xi}}{\gamma_{m}^{j} /(2 \varepsilon)+\xi} d \xi .
\end{aligned}
$$

To conclude the proof, it remains to evaluate the integral of the second term of the right hand side of the above equation. To this aim, we use standard complex analysis. Let us first notice that $\overline{\mathcal{F} U_{m}^{j}}$ can be extended to an holomorphic function for $z \in \mathbb{C}^{+}=\{z \in \mathbb{C}, \quad \operatorname{Im} z \geq 0\}$. Besides, we have the estimates, for $z=r e^{i \theta} \in \mathbb{C}^{+}$,

$$
\left|\overline{\mathcal{F} U_{m}^{j}}(z)\right| \leq \frac{C(\delta)}{(r \sin \theta)^{\frac{1+\delta}{2+\delta}}}\left\|u_{m}^{j}\right\|_{L^{2+\delta}(0, t)}
$$

as well as

$$
\left|\overline{\mathcal{F} U_{m}^{j}}(z)\right| \leq\left\|u_{m}^{j}\right\|_{L^{1}(0, t)} .
$$

The function $\sqrt{\xi}$ is also holomorphic on $\mathbb{C}^{+}-\{0\}$ (provided we choose the convenient branch) so that, defining a convenient contour $\mathrm{C}=\mathrm{C}_{R} \cup \mathrm{C}_{\eta_{1}} \cup \mathrm{C}_{\eta_{2}} \cup \mathrm{C}_{R, \eta_{1}, \eta_{2}} \subset \mathbb{C}^{+}$, 
where $C_{R}$ is the semi-circle centered at 0 of radius $R$, with $R>\left|\gamma_{m}^{j}\right| /(2 \varepsilon), C_{\eta_{1}}$ is the semi-circle centered at 0 of radius $\eta_{1}, C_{\eta_{2}}$ is the semi-circle centered at $-\gamma_{m}^{j} /(2 \varepsilon)$ of radius $\eta_{2}$, and $\mathrm{C}_{R, \eta_{1}, \eta_{2}}$ connects the different semi-circles on $\mathbb{R}$, the Cauchy theorem yields

$$
\int_{\mathrm{C}} \overline{\mathcal{F} U_{m}^{j}}(z) \frac{-\sqrt{z}}{\gamma_{m}^{j} /(2 \varepsilon)+z} d z=0
$$

We evaluate now the integral on each contour. We have, according to (3.19),

$$
\left|\int_{\mathrm{C}_{R}} \overline{\mathcal{F} U_{m}^{j}}(z) \frac{\bar{z}}{\gamma_{m}^{j} /(2 \varepsilon)+z} d z\right| \leq \frac{C}{R^{\delta /(2(2+\delta))}}\left\|u_{m}^{j}\right\|_{L^{2+\delta}(0, t)} \int_{0}^{\pi} \frac{d \theta}{|\sin \theta|^{\frac{1+\delta}{2+\delta}}},
$$

so that the integral on $C_{R}$ goes to zero as $R$ goes to the infinity as soon as $\delta>0$. In the same way, according to (3.20),

$$
\left|\int_{\mathrm{C}_{\eta_{1}}} \overline{\mathcal{F} U_{m}^{j}}(z) \frac{\sqrt{z}}{\gamma_{m}^{j} /(2 \varepsilon)+z} d z\right| \leq C \eta_{1}^{3 / 2}\left\|u_{m}^{j}\right\|_{L^{1}(0, t)},
$$

which vanishes in the limit $\eta_{1} \rightarrow 0$. Concerning the integral on $C_{\eta_{2}}$, we have

$$
\int_{\mathrm{C}_{\eta_{2}}} \overline{\mathcal{F} U_{m}^{j}}(z) \frac{-\sqrt{z}}{\gamma_{m}^{j} /(2 \varepsilon)+z} d z=\int_{\pi}^{0} \overline{\mathcal{F} U_{m}^{j}}\left(-\gamma_{m}^{j} /(2 \varepsilon)+\eta_{2} e^{i \theta}\right) \sqrt[-]{-\gamma_{m}^{j} /(2 \varepsilon)+\eta_{2} e^{i \theta}} i d \theta,
$$

so that the Lebesgue dominated convergence theorem implies that the integral goes to $-i \pi \sqrt{-\gamma_{m}^{j} /(2 \varepsilon)} \overline{\mathcal{F} U_{m}^{j}}\left(-\gamma_{m}^{j} /(2 \varepsilon)\right)$ as $\eta_{2} \rightarrow 0$. Since the integral on $C_{R, \eta_{1}, \eta_{2}}$ tends to

$$
\text { p.v. } \int_{\mathbb{R}} \overline{\mathcal{F} U_{m}^{j}}(\xi) \frac{-\sqrt{\xi}}{\gamma_{m}^{j} /(2 \varepsilon)+\xi} d \xi
$$

as $R \rightarrow \infty, \eta_{1} \rightarrow 0$ and $\eta_{2} \rightarrow 0$, we finally get, using (3.21),

$$
\text { p.v. } \int_{\mathbb{R}} \overline{\mathcal{F} U_{m}^{j}}(\xi) \frac{\overline{\sqrt{\xi}}}{\gamma_{m}^{j} /(2 \varepsilon)+\xi} d \xi=i \frac{\pi}{\sqrt{2 \varepsilon}} \overline{\sqrt{-\gamma_{m}^{j}}} \overline{\mathcal{F} U_{m}^{j}}\left(-\gamma_{m}^{j} /(2 \varepsilon)\right) .
$$

Consequently,

$$
\int_{0}^{t}\left\langle\mathbb{C}_{j}\left(\psi_{\lambda}^{p w}\right), u\right\rangle_{j} d s=\frac{1}{\varepsilon} \sum_{m} \sqrt{-\gamma_{m}^{j}} \psi_{m}^{0, j} \overline{\mathcal{F} U_{m}^{j}}\left(-\gamma_{m}^{j} /(2 \varepsilon)\right),
$$

and it remains to notice that $\sqrt{-\gamma_{m}^{j}}=-i \sqrt[+]{\gamma_{m}^{j}}$ to end the proof.

\section{Proof of the theorem}

We start by regularizing the problem to apply the previous existence result of [12] given in Theorem 3.1 where the initial condition is more regular. To be able to use $\psi_{\lambda}$ as a test function, we nevertheless need more regularity for $\psi_{\lambda}$ than that given in the theorem, which is only $\psi_{\lambda} \in \psi_{\lambda}^{p w}+\mathcal{C}^{0}\left([0, T], H^{2}(\Omega)\right) \cap \mathcal{C}^{1}\left([0, T], L^{2}(\Omega)\right)$. We thus consider a sequence of regular data $\psi_{\lambda}^{0, k} \in \mathcal{C}^{\infty}(\Omega)$ and $V_{e}^{k} \in \mathcal{C}^{\infty}([0, T] \Omega)$ such that (3.8)-(3.11) admits a unique solution $\psi_{\lambda}^{k}$ verifying $\psi_{\lambda}^{k} \in \mathcal{C}^{\infty}([0, T] \Omega)$. All the manipulations that will follow further are then justified. The regularization procedure 
is not direct and is sketched in Appendix B. The regularized data satisfies, for $1 \leq p<$ $\infty$

$$
\psi_{\lambda}^{0, k} \rightarrow \psi_{\lambda}^{0} \quad \text { strongly in } \quad H^{1}(K) ; \quad V_{e}^{k} \rightarrow V_{e} \quad \text { strongly in } \quad \mathcal{C}^{1}\left([0, T], L^{p}(K)\right),
$$

for any bounded set $K \subset \Omega$. This implies that, for any $u \in H^{1 / 4}\left((0, T), L^{2}\left(\Gamma_{j}\right)\right)$,

$$
\begin{aligned}
& \mathbb{D}_{j, k}^{1 / 2}(u) \rightarrow \mathbb{D}_{j}^{1 / 2}(u) \quad \text { strongly in } H^{-1 / 4}\left((0, T), L^{2}\left(\Gamma_{j}\right)\right), \\
& { }_{j, k}^{\varepsilon}\left(\psi_{\lambda}^{p w, k}\right) \rightarrow{ }_{j}^{\varepsilon}\left(\psi_{\lambda}^{p w}\right) \text { weakly in } L^{2}\left((0, T), L^{2}\left(\Gamma_{j}\right)\right) \text {. }
\end{aligned}
$$

In the same way, $\Phi(p, m, j)$ is localized so that the obtained $\Phi^{k}(p, m, j)$ has compact support and $\Phi^{k}$ converges strongly to $\Phi$ in $L^{1}\left(\mathbb{R}_{+}^{*}, \ell^{1}\left(\mathbb{N}^{*}\{1, \ldots, n\}\right)\right)$.

We prove now the bounds that will allow passing to the limit. They will also provide the $\varepsilon$-independent estimates.

Density estimate. Consider this regular solution $\psi_{\lambda}^{k}$ which is also obviously a solution to (3.13). Multiplying (3.8) by $\psi_{\lambda}^{k}$, integrating in $[0, T]$ and taking the imaginary part, it follows that

$$
\begin{gathered}
\left\|\psi_{\lambda}^{k}(T, \cdot)\right\|_{L^{2}\left(\Omega_{0}\right)}^{2}=\left\|\psi_{\lambda}^{0, k}\right\|_{L^{2}\left(\Omega_{0}\right)}^{2}+\sqrt{\varepsilon} \sum_{j=1}^{n} \mathcal{I} m e^{-i \pi / 4} \int_{0}^{T}\left\langle\mathbb{D}_{j, k}^{1 / 2}\left(\psi_{\lambda}^{k}\right), \psi_{\lambda}^{k}\right\rangle_{j} d s \\
-\varepsilon \sum_{j=1}^{n} \mathcal{I} m \int_{0}^{T}\left\langle{ }_{j, k}\left(\psi_{\lambda}^{p w, k}\right), \psi_{\lambda}^{k}\right\rangle_{j} d s .
\end{gathered}
$$

We drop in the sequel the $k$ superscript to clarify the notation keeping in mind until further notice that the wavefunctions we are manipulating are the regular solutions. Define now for any $s \in \mathbb{R}$,

$$
\Phi_{m, T}^{j}(s)=\left(\widetilde{\left.\mathcal{T}_{j} \psi_{\lambda}\right)_{m}}(s),\right.
$$

where $\mathcal{T}_{j}$ is the transformation previously introduced in $(3.14),\left(\mathcal{T}_{j} \psi_{\lambda}\right)_{m}(s)=$ $\left\langle\mathcal{T}_{j} \psi_{\lambda}\left(s, \eta_{j}=0, \cdot\right), \chi_{m}^{0, j}\right\rangle_{j}$, and $\sim$ is the extension by 0 outside $[0, T]$. Then, according to (A.1) of Lemma A.1,

$$
\operatorname{Im} 2 \pi e^{-i \pi / 4} \int_{0}^{T}\left\langle\mathbb{D}_{j}^{1 / 2}\left(\psi_{\lambda}\right), \psi_{\lambda}\right\rangle_{j} d s=-\sqrt{2} \sum_{m} \int_{\mathbb{R}^{-}} \sqrt{-\xi}\left|\mathcal{F} \Phi_{m, T}^{j}(\xi)\right|^{2} d \xi .
$$

The term involving ${ }_{j}^{\varepsilon}$ is treated using Lemma 3.2.1. Using the expression for $\mathbb{S}_{j}$ given by (2.5), and since the electrons are injected with a momentum $p$, in the lead $j_{0}$ and in the transversal mode $m_{0}$, we have $E(\lambda)=\frac{1}{2} p^{2}+E_{m_{0}}^{j_{0}}$, and therefore

$$
\int_{0}^{T}\left\langle{ }_{j}^{\varepsilon}\left(\psi_{\lambda}^{p w}\right), \psi_{\lambda}\right\rangle_{j} d s=-\delta_{j_{0}}^{j} \frac{2 i p}{\varepsilon} \int_{0}^{T} \theta_{\lambda}^{j_{0}}(s) \overline{\psi_{m_{0}}^{j_{0}}(s)} d s=-\delta_{j_{0}}^{j} \frac{2 i p}{\varepsilon} \overline{\mathcal{F}} \overline{\Phi_{m_{0}, T}^{j_{0}}}\left(p^{2} / 2 \varepsilon\right) .
$$

Gathering the previous estimates and integrating with respect to $\lambda$, we find that

$$
n(T)+\frac{\sqrt{2 \varepsilon}}{2 \pi} \sum_{j, m} \int_{\Lambda} \int_{\mathbb{R}_{-}} \sqrt{-\xi}\left|\mathcal{F} \Phi_{m, T}^{j}(\xi)\right|^{2} d \xi d \mu
$$




$$
\leq n(0)+2 \int_{\Lambda} p\left|\mathcal{F} \overline{\Phi_{m_{0}, T}^{j_{0}}}\left(p^{2} / 2 \varepsilon\right)\right| d \mu,
$$

which gives after an integration in time,

$$
\begin{aligned}
\|n\|_{L^{1}\left(0, T^{*}\right)}+\frac{\sqrt{2 \varepsilon}}{2 \pi} & \sum_{j, m} \int_{0}^{T^{*}} \int_{\Lambda} \int_{\mathbb{R}_{-}} \sqrt{-\xi}\left|\mathcal{F} \Phi_{m, T}^{j}(\xi)\right|^{2} d \xi d \mu d T \\
& \leq T^{*} n(0)+2 \int_{0}^{T^{*}} \int_{\Lambda} p\left|\mathcal{F} \overline{\Phi_{m_{0}, T}^{j_{0}}}\left(p^{2} / 2 \varepsilon\right)\right| d \mu d T .
\end{aligned}
$$

Energy estimate. We multiply now (3.8) by $\partial_{t} \psi_{\lambda}$. Taking the real part and integrating in time yield,

$$
\begin{aligned}
\frac{\varepsilon^{2}}{2}\left\|\nabla \psi_{\lambda}(T, \cdot)\right\|_{L^{2}\left(\Omega_{0}\right)}^{2}= & \frac{\varepsilon^{2}}{2}\left\|\nabla \psi_{\lambda}^{0}\right\|_{L^{2}\left(\Omega_{0}\right)}^{2}+\varepsilon^{2} \sum_{j=1}^{n} \mathcal{R} e \int_{0}^{T}\left\langle\frac{\partial \psi_{\lambda}}{\partial \eta_{j}}, \partial_{s} \psi_{\lambda}\right\rangle_{j} d s \\
& -\int_{0}^{T} \int_{\Omega_{0}} V(s, x) \partial_{s}\left|\psi_{\lambda}(s, x)\right|^{2} d x d s .
\end{aligned}
$$

We need to be a bit careful to extend $\partial_{s} \psi_{\lambda}$ by zero to $\mathbb{R}$ to use the Fourier tranform since $\psi_{\lambda}$ does not vanish at 0 and at $T$. Let $\widetilde{\psi_{\lambda}}$ be this extension and let $\partial_{s} \widetilde{\psi_{\lambda}}$ be its derivative in the distribution sense so that

$$
\frac{\partial \widetilde{\psi_{\lambda}}}{\partial s}(s, x)= \begin{cases}\frac{\partial \psi_{\lambda}}{\partial s}(s, x)-\psi_{\lambda}(T, x) \delta_{T}+\psi_{\lambda}(0, x) \delta_{0}, & s \in[0, T], \\ 0, & s \notin[0, T] .\end{cases}
$$

We then have

$$
\begin{aligned}
\int_{0}^{T}\left\langle\frac{\partial \psi_{\lambda}}{\partial \eta_{j}}, \partial_{s} \psi_{\lambda}\right\rangle_{j} d s= & \int_{0}^{T}\left\langle\frac{\partial \psi_{\lambda}}{\partial \eta_{j}}, \partial_{s} \widetilde{\psi_{\lambda}}\right\rangle_{j} d s \\
& +\left\langle\frac{\partial \psi_{\lambda}}{\partial \eta_{j}}, \psi_{\lambda}\right\rangle_{j}(T)-\left\langle\frac{\partial \psi_{\lambda}}{\partial \eta_{j}}, \psi_{\lambda}\right\rangle_{j}(0) .
\end{aligned}
$$

Using now the boundary conditions, both stationary and time-dependent, we find that

$$
\begin{aligned}
\int_{0}^{T}\left\langle\frac{\partial \psi_{\lambda}}{\partial \eta_{j}}, \partial_{s} \widetilde{\psi_{\lambda}}\right\rangle_{j} d s=-\frac{e^{-i \pi / 4}}{\sqrt{\varepsilon}} \int_{0}^{T}\left\langle\mathbb{D}_{j}^{1 / 2}\left(\psi_{\lambda}\right), \partial_{s} \widetilde{\psi_{\lambda}}\right\rangle_{j} d s+\int_{0}^{T}\left\langle{ }_{j}^{\varepsilon}\left(\psi_{\lambda}^{p w}\right), \partial_{s} \widetilde{\psi_{\lambda}}\right\rangle_{j} d s, \\
\left\langle\frac{\partial \psi_{\lambda}}{\partial \eta_{j}}, \psi_{\lambda}\right\rangle_{j}(T)=-\frac{e^{-i \pi / 4}}{\sqrt{\varepsilon}}\left\langle\mathbb{D}_{j}^{1 / 2}\left(\psi_{\lambda}\right), \psi_{\lambda}\right\rangle_{j}(T)+\left\langle{ }_{j}^{\varepsilon}\left(\psi_{\lambda}^{p w}\right), \psi_{\lambda}\right\rangle_{j}(T), \\
\left\langle\frac{\partial \psi_{\lambda}}{\partial \eta_{j}}, \psi_{\lambda}\right\rangle_{j}(0)=\frac{1}{\varepsilon}\left\langle\mathbb{Z}_{j}[E(\lambda)]\left(\psi_{\lambda}^{0}\right), \psi_{\lambda}^{0}\right\rangle_{j}+\frac{1}{\varepsilon}\left\langle\mathbb{S}_{j}[E(\lambda)], \psi_{\lambda}^{0}\right\rangle_{j},
\end{aligned}
$$

and we recast the energy relation as

$$
\begin{aligned}
\frac{\varepsilon^{2}}{2}\left\|\nabla \psi_{\lambda}(T, \cdot)\right\|_{L^{2}\left(\Omega_{0}\right)}^{2}+L_{1}+L_{2}= & \frac{\varepsilon^{2}}{2}\left\|\nabla \psi_{\lambda}^{0}\right\|_{L^{2}\left(\Omega_{0}\right)}^{2}+R_{1}+R_{2}+R_{3} \\
& -\int_{0}^{T} \int_{\Omega_{0}} V(s, x) \partial_{s}\left|\psi_{\lambda}(s, x)\right|^{2} d x d s
\end{aligned}
$$


where

$$
\begin{aligned}
& L_{1}=\varepsilon^{3 / 2} \sum_{j=1}^{n} \mathcal{R} e e^{-i \pi / 4} \int_{0}^{T}\left\langle\mathbb{D}_{j}^{1 / 2}\left(\psi_{\lambda}\right), \partial_{s} \widetilde{\psi_{\lambda}}\right\rangle_{j} d s, \\
& L_{2}=\varepsilon^{3 / 2} \sum_{j=1}^{n} \mathcal{R} e e^{-i \pi / 4}\left\langle\mathbb{D}_{j}^{1 / 2}\left(\psi_{\lambda}\right), \psi_{\lambda}\right\rangle_{j}(T), \\
& R_{1}=\varepsilon^{2} \sum_{j=1}^{n} \mathcal{R} e \int_{0}^{T}\left\langle\varepsilon_{j}^{\varepsilon}\left(\psi_{\lambda}^{p w}\right), \partial_{s} \widetilde{\psi_{\lambda}}\right\rangle_{j} d s, \\
& R_{2}=\varepsilon^{2} \sum_{j=1}^{n} \mathcal{R e}\left\langle{ }_{j}^{\varepsilon}\left(\psi_{\lambda}^{p w}\right), \psi_{\lambda}\right\rangle_{j}(T), \\
& R_{3}=-\varepsilon \sum_{j=1}^{n} \operatorname{Re}\left[\left\langle\mathbb{Z}_{j}[E(\lambda)]\left(\psi_{\lambda}^{0}\right), \psi_{\lambda}^{0}\right\rangle_{j}+\left\langle\mathbb{S}_{j}[E(\lambda)], \psi_{\lambda}^{0}\right\rangle_{j}\right] .
\end{aligned}
$$

We now treat each term separately. For the nonlinear boundary term $L_{1}$, we have, thanks to the Fourier-Plancherel equality:

$$
\begin{aligned}
& \operatorname{Re} 2 \pi e^{-i \pi / 4} \int_{0}^{T}\left\langle\mathbb{D}_{j}^{1 / 2}\left(\psi_{\lambda}\right), \partial_{s} \widetilde{\psi_{\lambda}}\right\rangle_{j} d s \\
= & \sqrt{2} \sum_{m} \int_{\mathbb{R}^{+}} \xi^{3 / 2}\left|\mathcal{F} \Phi_{m, T}^{j}(-\xi)\right|^{2} d \xi \\
-\frac{\sqrt{2}}{\varepsilon} & \mathcal{I} m 2 \pi e^{-i \pi / 4} \sum_{m} \int_{0}^{T}\left(E_{m}^{j}+V_{j}\right) \partial^{1 / 2}\left(\mathcal{T}_{j} \psi_{\lambda}\right)_{m} \overline{\left(\mathcal{T}_{j} \psi_{\lambda}\right)_{m}} d s .
\end{aligned}
$$

Using (4.4) and the fact that we assumed $E_{m}^{j} \geq 0, m \geq 1, j \geq 1$, we have:

$$
\begin{aligned}
& \operatorname{Im} 2 \pi e^{-i \pi / 4} \sum_{m} E_{m}^{j} \int_{0}^{T} \partial^{1 / 2}\left(\mathcal{T}_{j} \psi_{\lambda}\right)_{m} \overline{\left(\mathcal{T}_{j} \psi_{\lambda}\right)_{m}} d s \\
= & -\sqrt{2} \sum_{m} E_{m}^{j} \int_{\mathbb{R}^{-}} \sqrt{-\xi}\left|\mathcal{F} \Phi_{m, T}^{j}(\xi)\right|^{2} d \xi \leq 0 .
\end{aligned}
$$

The last term of $L_{1}$ is treated by multiplying the Schrdinger equation by $\sum_{j} \mu_{j} V_{j} \overline{\psi_{\lambda}}:=g \overline{\psi_{\lambda}}$, integrating in time and taking the imaginary part, which yield

$$
\begin{aligned}
& \left(g \psi_{\lambda}, \psi_{\lambda}\right)(T) \\
= & \left(g \psi_{\lambda}^{0}, \psi_{\lambda}^{0}\right)+\int_{0}^{T}\left(\partial_{s} g \psi_{\lambda}, \psi_{\lambda}\right)(s) d s+\mathcal{I} m \int_{0}^{T}\left(\nabla g, \overline{\psi_{\lambda}} \nabla \psi_{\lambda}\right)(s) d s \\
& +\mathcal{I} m \sum_{j=1}^{n}\left[\varepsilon^{1 / 2} e^{-i \pi / 4} \int_{0}^{T}\left\langle\mathbb{D}_{j}^{1 / 2}\left(\psi_{\lambda}\right), V_{j} \psi_{\lambda}\right\rangle_{j} d s-\varepsilon \int_{0}^{T}\left\langle{ }_{j}^{\varepsilon}\left(\psi_{\lambda}^{p w}\right), V_{j} \psi_{\lambda}\right\rangle d s\right] .
\end{aligned}
$$

Applying (3.2.1) and using the expression of $\mathbb{S}_{j}$, we have

$$
\varepsilon\left|\sum_{j=1}^{n} \int_{0}^{T} \int_{\Lambda}\left\langle{ }_{j}^{\varepsilon}\left(\psi_{\lambda}^{p w}\right), V_{j} \psi_{\lambda}\right\rangle d s d \mu\right| \leq 2 \int_{\Lambda} p\left|\mathcal{F} V_{j_{0}} \overline{\Phi_{m_{0}, T}^{j_{0}}}\left(p^{2} / 2 \varepsilon\right)\right| d \mu .
$$

This finally gives for $L_{1}$,

$$
\frac{\sqrt{2}}{2 \pi} \sum_{m, j} \int_{\Lambda} \int_{\mathbb{R}^{+}} \varepsilon^{3 / 2} \xi^{3 / 2}\left|\mathcal{F} \Phi_{m, T}^{j}(-\xi)\right|^{2} d \xi d \mu+R_{1}^{1} \leq \int_{\Lambda} L_{1} d \mu
$$


where $R_{1}^{1}$ satisfies, using (4.5):

$$
\left|R_{1}^{1}\right| \leq C \int_{\Lambda} p\left|\mathcal{F} V_{j_{0}} \overline{\Phi_{m_{0}, T}^{j_{0}}}\left(p^{2} / 2 \varepsilon\right)\right| d \mu+C\left(n(0)+\|n\|_{L^{1}(0, T)}+\|\mathcal{E}\|_{L^{1}(0, T)}\right) .
$$

The generic constant $C$ above depends on $\left\|V_{j}\right\|_{\mathcal{C}^{1}([0, T])}$. For the boundary term $R_{1}$, an integration by parts and (3.2.1) give

$$
\begin{aligned}
R_{1} & =-\operatorname{Re} 2 i p \varepsilon \int_{0}^{T} \theta_{\lambda}^{j_{0}}(s) \overline{\partial_{t} \overline{\psi_{m_{0}}^{j_{0}}(s)}} d s \\
& =-2 p \operatorname{Re}\left[\frac{p^{2}}{2} \mathcal{F} \overline{\Phi_{m_{0}, T}^{j_{0}}}\left(p^{2} / 2 \varepsilon\right)+\mathcal{F} V_{j_{0}} \overline{\Phi_{m_{0}, T}^{j_{0}}}\left(p^{2} / 2 \varepsilon\right)\right] .
\end{aligned}
$$

The term $L_{2}$ is integrated in time to be able to use similar arguments as those of the density estimate. This yields

$$
\int_{0}^{T^{*}} L_{2}(T) d T=\frac{\sqrt{2} \varepsilon^{3 / 2}}{2 \pi} \sum_{j, m} \int_{\mathbb{R}_{+}} \sqrt{\xi}\left|\mathcal{F} \Phi_{m, T^{*}}^{j}(\xi)\right|^{2} d \xi
$$

and in the same way,

$$
\int_{0}^{T^{*}} R_{2}(T) d T=\varepsilon 2 p \mathcal{I m} \mathcal{F} \overline{\Phi_{m_{0}, T^{*}}^{j_{0}}}\left(p^{2} / 2 \varepsilon\right)
$$

The last term $R_{3}$ is straightforwardly bounded thanks to hypothesis (3.16) and Lemma A.1:

$$
\left|\int_{\Lambda} R_{3} d \mu\right| \leq C_{0}+C\|p\|_{L^{2}(\Lambda ; d \mu)}^{1 / 2} n^{1 / 4}(0) \mathcal{E}^{1 / 4}(0)
$$

It remains to tackle the term involving the potential $V=V_{e}+V_{s}$. Using the Poisson equation (3.11), we find that

$$
\int_{0}^{T} \int_{\Lambda} \int_{\Omega_{0}} V_{s} \partial_{s}\left|\psi_{\lambda}\right|^{2} d x d s d \mu=\frac{1}{2}\left\|\nabla V_{s}(T, \cdot)\right\|_{L^{2}\left(\Omega_{0}\right)}^{2}-\frac{1}{2}\left\|\nabla V_{s}(0, \cdot)\right\|_{L^{2}\left(\Omega_{0}\right)}^{2} .
$$

The term including the exterior potential $V_{e}$ is easily handled after an integration by parts,

$$
\left.\left|\int_{\Lambda} \int_{0}^{T} \int_{\Omega_{0}} V_{e} \partial_{s}\right| \psi_{\lambda}\right|^{2} d x d s d \mu \mid \leq\left\|V_{e}\right\|_{\mathcal{C}^{1}\left([0, T], L^{\infty}\left(\Omega_{0}\right)\right)}\left(n(T)+n(0)+\int_{0}^{T} n(s) d s\right) .
$$

The next step is to integrate (4.7) with respect to $\lambda$, to add (4.6) to it and to use (4.8), (4.10), (4.9), (4.11), (4.12) and hypothesis (3.16). Gathering the different estimates, we find that

$$
\|\mathcal{E}\|_{L^{1}\left(0, T^{*}\right)}+\|n\|_{L^{1}\left(0, T^{*}\right)}+T_{4} \leq C_{1}+R_{4}+C_{2} \int_{0}^{T^{*}}\left(\|n\|_{L^{1}(0, T)}+\|\mathcal{E}\|_{L^{1}(0, T)}\right) d T,
$$


where

$$
\begin{aligned}
T_{4}= & \frac{\sqrt{2} \varepsilon^{3 / 2}}{2 \pi} \sum_{j, m} \int_{0}^{T^{*}} \int_{\Lambda} \int_{\mathbb{R}^{+}} \xi^{3 / 2}\left|\mathcal{F} \Phi_{m, T}^{j}(-\xi)\right|^{2} d \xi d \mu d T \\
& +\frac{\sqrt{2 \varepsilon}}{2 \pi} \sum_{j, m} \int_{0}^{T^{*}} \int_{\Lambda} \int_{\mathbb{R}_{-}} \sqrt{-\xi}\left|\mathcal{F} \Phi_{m, T}^{j}(\xi)\right|^{2} d \xi d \mu d T \\
& +\frac{\sqrt{2} \varepsilon^{3 / 2}}{2 \pi} \sum_{j, m} \int_{\mathbb{R}_{+}} \sqrt{\xi}\left|\mathcal{F} \Phi_{m, T^{*}}^{j}(\xi)\right|^{2} d \xi d \mu, \\
R_{4}= & \int_{0}^{T^{*}} \int_{\Lambda} p^{3}\left|\mathcal{F} \overline{\Phi_{m_{0}, T}^{j_{0}}}\left(p^{2} / 2 \varepsilon\right)\right| d \mu d T+C_{3} \int_{0}^{T^{*}} \int_{\Lambda} p\left|\mathcal{F} V_{j_{0}} \overline{\Phi_{m_{0}, T}^{j_{0}}}\left(p^{2} / 2 \varepsilon\right)\right| d \mu d T \\
& +2 \varepsilon \int_{\Lambda} p\left|\overline{\mathcal{F}} \overline{\Phi_{m_{0}, T^{*}}^{j_{0}}}\left(p^{2} / 2 \varepsilon\right)\right| d \mu+C_{4} \int_{0}^{T^{*}} \int_{\Lambda} p\left|\mathcal{F} \overline{\Phi_{m_{0}, T}^{j_{0}}}\left(p^{2} / 2 \varepsilon\right)\right| d \mu d T .
\end{aligned}
$$

The constants $C_{1}, C_{2}, C_{3}$ and $C_{4}$ depend on the constant $C_{0}$ of (3.16) and on $\left\|V_{e}\right\|_{\mathcal{C}^{1}\left([0, T], L^{\infty}(\Omega)\right)}$. To end the proof we need now to bound $R_{4}$ and thus $\mathcal{F} \overline{\Phi_{m_{0}, t}^{j_{0}}}\left(p^{2} / \varepsilon\right)$, for $t=T, T^{*}$. A straightforward attempt by using trace theorems would again fail because of the homogeneity in $\varepsilon$. Indeed, we expect to estimate the trace with the help of the derivative of $\psi_{\lambda}$, while the energy involves a derivative multiplied by $\varepsilon$. Nevertheless, it is expected that $\overline{\mathcal{F}} \overline{\Phi_{m_{0}, t}^{j_{0}}}\left(p^{2} / \varepsilon\right)$ goes to zero as $\varepsilon \rightarrow 0$, and the rate of convergence is given by the regularity in time of $\psi_{\lambda}$, which induces the decay of $\mathcal{F} \overline{\Phi_{m_{0}, t}^{j_{0}}}(\xi)$. This will allow to recover a correct homogeneity in $\varepsilon$ to be able to use trace theorems. To this aim, we apply Lemma (A.2), which provides, for $p>0$,

$$
\left|\mathcal{F} \overline{\Phi_{m_{0}, t}^{j_{0}}}\left(p^{2} / 2 \varepsilon\right)\right| \leq C\left(\frac{\varepsilon}{p^{2}}\right)^{1 / 4}\left\|\xi^{1 / 4} \mathcal{F} \overline{\Phi_{m_{0}, t}^{j_{0}}}\right\|_{L^{2}\left(\mathbb{R}^{+}\right)}+C\left(\frac{\varepsilon}{p^{2}}\right)^{1 / 2}\left\|\mathcal{F} \overline{\Phi_{m_{0}, t}^{j_{0}}}\right\|_{L^{2}(\mathbb{R})}
$$

and Lemma (A.1) yields

$$
\begin{aligned}
\left\|\mathcal{F} \overline{\Phi_{m_{0}, t}^{j_{0}}}\right\|_{L^{2}(\mathbb{R})}=2 \pi\left\|\psi_{m_{0}, t}^{j_{0}}\right\|_{L^{2}(0, t)} & \leq 2 \pi\left\|\psi_{\lambda}\right\|_{L^{2}\left((0, t), L^{2}\left(\Gamma_{j_{0}}\right)\right)} \\
& \leq C\left\|\psi_{\lambda}\right\|_{L^{2}\left((0, t), L^{2}\left(\Omega_{0}\right)\right)}^{1 / 2}\left\|\nabla \psi_{\lambda}\right\|_{L^{2}\left((0, t), L^{2}\left(\Omega_{0}\right)\right)}^{1 / 2} .
\end{aligned}
$$

Notice here the fact that the $L^{2}$ norm of the trace is controlled by $\left\|\nabla \psi_{\lambda}\right\|_{L^{2}\left((0, t), L^{2}\left(\Omega_{0}\right)\right)}^{1 / 2}$ while with non-flat interfaces the exponent would have been $1 / 2+\alpha$, with $\alpha>0$. This would have led to a wrong homogeneity. We thus have, for $\beta=1,3$,

$$
\begin{aligned}
\int_{\Lambda} p^{\beta}\left|\mathcal{F} \overline{\Phi_{m_{0}, t}^{j_{0}}}\left(p^{2} / 2 \varepsilon\right)\right| d \mu \leq C\|n\|_{L^{1}(0, t)}^{1 / 2} & +C\|\mathcal{E}\|_{L^{1}(0, t)}^{1 / 2} \\
& +C \varepsilon^{1 / 4}\left(\int_{\Lambda}\left\|\xi^{1 / 4} \mathcal{F} \overline{\Phi_{m_{0}, t}^{j_{0}}}\right\|_{L^{2}\left(\mathbb{R}^{+}\right)}^{2} d \mu\right)^{1 / 2}
\end{aligned}
$$

where the constant $C$ depends on $\|p\|_{L^{5}(\Lambda, d \mu)}$. According to Rem. A.3, it can be easily shown that we have the same estimate as above for $\int_{\Lambda} p\left|\mathcal{F} V_{j_{0}} \overline{\Phi_{m_{0}, t}^{j_{0}}}\left(p^{2} / 2 \varepsilon\right)\right| d \mu$. We now control the last term of the right hand side for any $\eta>0$,

$$
\varepsilon^{1 / 4}\left(\int_{\Lambda}\left\|\xi^{1 / 4} \mathcal{F} \overline{\Phi_{m_{0}, t}^{j_{0}}}\right\|_{L^{2}\left(\mathbb{R}^{+}\right)}^{2} d \mu\right)^{1 / 2} \leq \frac{1}{\eta}+\eta \sqrt{\varepsilon} \sum_{j, m} \int_{\Lambda} \int_{\mathbb{R}^{-}} \sqrt{-\xi}\left|\mathcal{F} \Phi_{m, t}^{j}(\xi)\right|^{2} d \xi d \mu .
$$


This allows to write

$$
R_{4} \leq C_{\eta}+C \eta\left(\|n\|_{L^{1}\left(0, T^{*}\right)}+\|\mathcal{E}\|_{L^{1}\left(0, T^{*}\right)}+T_{4}\right),
$$

so that the Gronwall lemma and (4.13) imply that $\|n\|_{L^{1}\left(0, T^{*}\right)},\|\mathcal{E}\|_{L^{1}\left(0, T^{*}\right)}$ are bounded independently of $\varepsilon$ for any finite $T^{*}>0$ and in turn so are $T_{4}$ and $\|n\|_{L^{\infty}\left(0, T^{*}\right)}$ thanks to (4.5). In particular, we obtain that

$$
\sqrt{\varepsilon} \sum_{j, m} \int_{\Lambda} \int_{\mathbb{R}} \sqrt{\xi}\left|\mathcal{F} \Phi_{m, T^{*}}^{j}(\xi)\right|^{2} d \xi d \mu \leq C,
$$

where $C$ is independent of $\varepsilon$ and of the regularization parameter $k$, and thus a bound in $H^{1 / 4}\left((0, T), L^{2}\left(\Gamma_{j}\right)\right)$ for $\mathcal{T}_{j} \psi_{\lambda}$.

To conclude the derivation of the different bounds, it remains to estimate $V_{s}$. The fact that $\|n\|_{L^{\infty}(0, T)}$ and $\|\mathcal{E}\|_{L^{1}(0, T)}$ are bounded imply that $\int_{\Lambda}\left|\psi_{\lambda}\right|^{2} d \mu(\lambda) \in$ $L^{2}\left((0, T), W^{1, r}\left(\Omega_{0}\right)\right)$, with $r<2$ for $d=2$ and $r=\frac{3}{2}$ for $d=3$, which in turn gives $V_{s} \in L^{2}\left((0, T), W^{3, r}\left(\Omega_{0}\right)\right)$, thanks to standard elliptic regularity results. We estimate now $\partial_{t} V_{s}$, which solves

$$
\Delta \partial_{t} V_{s}=J, \quad x \in \Omega_{0}, \quad \partial_{t} V_{s}=0, \quad x \in \partial \Omega_{0},
$$

where $J=\mathcal{I} m \varepsilon \int_{\Lambda} \bar{\psi}_{\lambda} \nabla \psi_{\lambda} d \mu(\lambda) . \quad J$ belongs to $L^{2}\left((0, T), L^{r}\left(\Omega_{0}\right)\right)$, with the same $r$ as above. Elliptic regularity then implies that $\partial_{t} V_{s}$ is in $L^{2}\left((0, T), W^{1, r}\left(\Omega_{0}\right)\right)$. To get the $\varepsilon$-independent bound on $\partial_{t} V_{s}$, we cannot use any Sobolev embeddings such that $J$ is bounded independently of $\varepsilon$ only in $L^{2}\left((0, T), L^{1}\left(\Omega_{0}\right)\right)$, which implies the $L^{2}\left((0, T), L^{r}\left(\Omega_{0}\right)\right)$ estimate announced in $(3.17)$.

End of the proof of existence. So far, estimate (3.17) has been proved for regular data. It remains true at the limit for unregularized data as we will see in the sequel using standard compactness results. Thanks to (3.17),

$$
\left.\psi_{\lambda}^{k} \in L^{\infty}\left((0, T), L^{2}\left(\Omega_{0} \Lambda ; d x d \mu^{k}\right)\right) ; \quad \nabla \psi_{\lambda}^{k} \in L^{2}\left((0, T), L^{2}\left(\Omega_{0} \Lambda ; d x d \mu^{k}\right)\right)\right),
$$

with bounds independent of $k$, so that $\psi_{\lambda}^{k}$ converges weakly- ${ }^{*}$ to a limit $\psi_{\lambda} \in$ $L^{\infty}\left((0, T), L^{2}\left(\Omega_{0} \Lambda ; d x d \mu\right)\right)$ as well as for $\nabla \psi_{\lambda}^{k}$, up to the extraction of a subsequence. Remember that $\Phi^{k}$ converges strongly to $\Phi$ in $L^{1}\left(\mathbb{R}_{+}, \ell^{1}\left(\mathbb{N}^{*}\{1, \ldots, n\}\right)\right)$, so that the weak-* limit of $\psi_{\lambda}^{k}$ in $L^{\infty}\left((0, T), L^{2}\left(\Omega_{0} \Lambda ; d x d \mu^{k}\right)\right)$ is equal to that of $\psi_{\lambda}^{k}$ in $L^{\infty}\left((0, T), L^{2}\left(\Omega_{0} \Lambda ; d x d \mu\right)\right)$. This implies that

$$
\left\|\psi_{\lambda}\right\|_{L^{\infty}\left((0, T), L^{2}\left(\Omega_{0} \Lambda ; d x d \mu\right)\right.} \leq \liminf \left\|\psi_{\lambda}^{k}\right\|_{L^{\infty}\left((0, T), L^{2}\left(\Omega_{0} \Lambda ; d x d \mu^{k}\right)\right.},
$$

with the same relation for $\nabla \psi_{\lambda}$ with an $L^{2}$ norm in time. Hence, estimate (3.17) is also verified at the limit by $\psi_{\lambda}$ and $\nabla \psi_{\lambda}$.

We pass now to the limit in the weak formulation $(3.13) . \quad \psi_{\lambda}^{k}$ is obviously a solution to (3.13). Integrating with respect to $\Lambda$ and choosing a test function $u$ such that $u \in \mathcal{C}^{1}\left([0, T), L^{2}\left(\Omega_{0} \Lambda ; d x d \mu\right)\right)$ and $\nabla u \in \mathcal{C}^{1}\left([0, T), L^{2}\left(\Omega_{0} \Lambda ; d x d \mu\right)\right)$, the nonboundary linear terms pass to the limit readily; only the boundary terms and the non-linear one require some attention. Thanks to the previously obtained bounds, we have for $d=2$ or $d=3$,

$$
V_{s}^{k} \in L^{2}\left((0, T), H_{0}^{1}\left(\Omega_{0}\right)\right) ; \quad \partial_{t} V_{s}^{k} \in L^{2}\left((0, T), L^{r}\left(\Omega_{0}\right)\right),
$$


where $r<2$ when $d=2$ and $r<\frac{3}{2}$ when $d=3$ with bounds independent of $k$, so that there still exists a subsequence - denoted by $V_{s}^{k}$ - such that $V_{s}^{k}$ converges strongly to $V_{s}$ in $L^{2}\left((0, T), L^{r}\left(\Omega_{0}\right)\right)$, for any $1 \leq r \leq 6$ in three dimensions and for any $1 \leq r<\infty$ in two dimensions. See [31] for a standard compactness result. In the same way, thanks to standard Sobolev embeddings, $\psi_{\lambda}^{k}$ converges weakly - up to the extraction of a subsequence - to $\psi_{\lambda}$ in $L^{2}\left((0, T), L^{r, 2}\left(\Omega_{0} \Lambda ; d x d \mu\right)\right.$ ) (where $L^{r, 2}\left(\Omega_{0} \Lambda ; d x d \mu\right)$ is defined as the Banach space of functions $f$ such that $\left.\int_{\Omega_{0}}\|f(x, \cdot)\|_{L^{2}(\Lambda, d \mu)}^{r} d x<\infty\right)$, for any $1 \leq r \leq 6$ in three dimensions and for any $1 \leq r<\infty$ in two dimensions. This thus allows to pass to the limit in the non-linear term

$$
\int_{0}^{T} \int_{\Lambda} \int_{\Omega_{0}} V_{s}^{k}(s, x) \psi_{\lambda}^{k}(s, x) u(s, x, \lambda) d x d s d \mu .
$$

The boundary terms remain, namely

$$
\frac{1}{2} \sum_{j=1}^{n}\left[\varepsilon^{1 / 2} e^{-i \pi / 4} \int_{0}^{T}\left\langle\mathbb{D}_{j, k}^{1 / 2}\left(\psi_{\lambda}\right), u\right\rangle_{j} d s+\varepsilon \int_{0}^{T}\left\langle{ }_{j, k}^{\varepsilon}\left(\psi_{\lambda}^{p w}\right), u\right\rangle d s\right] .
$$

The second term converges straightforwardly because of (4.3). The first one is treated thanks to its weak formulation (3.15). We know from (4.15) that $\mathcal{T}_{j} \psi_{\lambda}^{k}$ is bounded in $H^{1 / 4}\left((0, T), L^{2}\left(\Gamma_{j}\right)\right)$, independently of $k$ so that thanks to $(4.2), \int_{0}^{T}\left\langle\mathbb{D}_{j, k}^{1 / 2}\left(\psi_{\lambda}^{k}\right), u\right\rangle_{j} d s$ converges to $\int_{0}^{T}\left\langle\mathbb{D}_{j}^{1 / 2}\left(\psi_{\lambda}\right), u\right\rangle_{j} d s$.

We show now that the limit $\psi_{\lambda}$ actually belongs to $\mathcal{C}^{0}\left([0, T], L^{2}\left(\Omega_{0}\right)\right), \lambda$ a.e., so that the initial condition can be identified. Let us denote by $\psi_{\lambda}^{k}$ and $\psi_{\lambda}^{l}, k, l \in \mathbb{N}^{*}$, two elements of the sequence of regularized solutions and define $w=\psi_{\lambda}^{k}-\psi_{\lambda}^{l}$. w verifies the following boundary conditions on $\Gamma_{j}$,

$$
\frac{\partial w}{\partial \eta_{j}}=-\frac{e^{-i \pi / 4}}{\sqrt{\varepsilon}} \mathbb{D}_{j, k}^{1 / 2}(w)+\frac{e^{-i \pi / 4}}{\sqrt{\varepsilon}}\left[\mathbb{D}_{j, k}^{1 / 2}-\mathbb{D}_{j, l}^{1 / 2}\right]\left(\psi_{\lambda}^{l}\right)+\left[\varepsilon_{j, k}\left(\psi_{\lambda}^{p w, k}\right)-{ }_{j, l}^{\varepsilon}\left(\psi_{\lambda}^{p w, l}\right)\right] .
$$

Proceeding as in the proof of estimate (4.5), we obtain

$$
\begin{aligned}
\|w(t, \cdot)\|_{L^{2}\left(\Omega_{0}\right)}^{2}= & \|w(t=0, \cdot)\|_{L^{2}\left(\Omega_{0}\right)}^{2}+\sqrt{\varepsilon} \sum_{j=1}^{n} \mathcal{I} m e^{-i \pi / 4} \int_{0}^{t}\left\langle\mathbb{D}_{j, k}^{1 / 2}(w), w\right\rangle_{j} d s \\
& +\frac{2}{\varepsilon} \mathcal{I} m \int_{0}^{t} \int_{\Omega_{0}}\left(V^{k}-V^{l}\right) \psi_{\lambda}^{l} \bar{w} d x d s+R_{1}+R_{2}, \\
R_{1}= & -\sqrt{\varepsilon} \sum_{j=1}^{n} \mathcal{I} m e^{-i \pi / 4} \int_{0}^{t}\left\langle\left[\mathbb{D}_{j, k}^{1 / 2}-\mathbb{D}_{j, l}^{1 / 2}\right]\left(\psi_{\lambda}^{l}\right), w\right\rangle_{j} d s, \\
R_{2}= & -\varepsilon \sum_{j=1}^{n} \mathcal{I m} \int_{0}^{t}\left\langle{ }_{j, k}\left(\psi_{\lambda}^{p w, k}\right)-{ }_{j, l}^{\varepsilon}\left(\psi_{\lambda}^{p w, l}\right), w\right\rangle_{j} d s .
\end{aligned}
$$

The first term of the right hand side goes to zero as $k, l \rightarrow \infty$ thanks to (4.1); the second one is negative; the third one is treated using the Gronwall Lemma and that

$$
\begin{aligned}
\left\|\left(\left(V_{s}^{k}-V_{s}^{l}\right) \psi_{\lambda}^{l}\right)(t, \cdot)\right\|_{L^{2}\left(\Omega_{0} \Lambda ; d x d \mu\right)} & \leq\left\|\left(V_{s}^{k}-V_{s}^{l}\right)(t, \cdot)\right\|_{L^{3}\left(\Omega_{0}\right)}\left\|\psi_{\lambda}^{l}(t, \cdot)\right\|_{L^{6}\left(\Omega_{0}, L^{2}(\Lambda, d \mu)\right)} \\
& \leq C \mathcal{E}(t)\|w(t, \cdot)\|_{L^{2}\left(\Omega_{0} \Lambda ; d x d \mu\right) .}
\end{aligned}
$$


Moreover $R_{1}, R_{2}$ converge to zero as well thanks to (4.2), (4.3) and (4.15). $\psi_{\lambda}^{k}-\psi_{\lambda}^{l}$ is thus a Cauchy sequence in $\mathcal{C}^{0}\left([0, T], L^{2}\left(\Omega_{0}\right)\right)$, so that the limit $\psi_{\lambda}$ belongs as well to this space.

It just remains to show that (3.13) indeed holds almost everywhere in $\lambda$ and to treat the Poisson equation. For the first claim, it suffices to take a test function of the form $u(t, x) f(\lambda)$, with $u \in \mathcal{C}^{1}\left([0, T), H^{1}\left(\Omega_{0}\right)\right), f \in L^{2}(\Lambda)$. For the Poisson equation, using a weak formulation, $V_{s}^{k}$ passes straightforwardly to the limit, as well as $\int_{\Lambda}\left|\psi_{\lambda}^{k}\right|^{2} d \mu$. We only need to show that its limit equals $\int_{\Lambda}\left|\psi_{\lambda}\right|^{2} d \mu$. This requires some local estimates with respect to $\lambda$. Using the fact that the non-linear potential $V_{s}^{k}$ is bounded, the density and energy estimates can be rewritten in local in $\lambda$ versions so as to obtain

$$
\left\|\psi_{\lambda}^{k}\right\|_{L^{2}\left((0, T), H^{1}\left(\Omega_{0}\right)\right)}^{2} \leq C(T)\left(1+p^{5}+\left\|\psi_{\lambda}^{0}\right\|_{\left.L^{2}\left(\Omega_{0}\right)\right)}^{2}+\varepsilon^{2}\left\|\nabla \psi_{\lambda}^{0}\right\|_{L^{2}\left(\Omega_{0}\right)}^{2}\right),
$$

for some constant $C$ independent of $k$. Multiplying by $\Phi$, and using (2.2) and hypothesis (H-3), we get a uniform in $\lambda$ bound for $\Phi\left\|\psi_{\lambda}^{k}\right\|_{L^{2}\left((0, T), H^{1}\left(\Omega_{0}\right)\right)}^{2}$. We thus have that $\sqrt{\Phi} \psi_{\lambda}^{k}$ converges weakly - up to the extraction of a subsequence - in $L^{2}\left((0, T), L^{r}\left(\Omega_{0}\right)\right)$ to $\sqrt{\Phi} \psi_{\lambda}, \lambda$ a.e., where $r \leq 6$ in three dimensions and $r<\infty$ in two dimensions. This allows then to identify the limit of $\int_{\Lambda}\left|\psi_{\lambda}^{k}\right|^{2} d \mu$ with $\int_{\Lambda}\left|\psi_{\lambda}\right|^{2} d \mu$ and concludes the proof of existence.

Uniqueness. We claim that two solutions to (3.13) in $\mathrm{E}^{0}, \psi_{\lambda}^{i}, i=1,2$, with the same initial condition satisfy the relation

$$
\left\|\psi_{\lambda}^{1}-\psi_{\lambda}^{2}\right\|_{L^{2}\left(\Omega_{0}\right)}^{2} \leq \frac{2}{\varepsilon} \mathcal{I} m \int_{0}^{t} \int_{\Omega_{0}}\left(V_{s}^{1}-V_{s}^{2}\right) \psi_{\lambda}^{1}\left(\overline{\psi_{\lambda}^{1}}-\overline{\psi_{\lambda}^{2}}\right) d x d s .
$$

Such an estimate is proved in the same manner as the density estimate (4.6): by first deriving it with regular solutions, and then by passing to the limit. Following (4.16), we obtain

$$
\left\|\left(\psi_{\lambda}^{1}-\psi_{\lambda}^{2}\right)(t, \cdot)\right\|_{L^{2}\left(\Omega_{0} \Lambda ; d x d \mu\right)} \leq C \int_{0}^{t} \mathcal{E}(s)\left\|\left(\psi_{\lambda}^{1}-\psi_{\lambda}^{2}\right)(s, \cdot)\right\|_{L^{2}\left(\Omega_{0} \Lambda ; d x d \mu\right)} d s,
$$

so that the Gronwall Lemma yields $\psi_{\lambda}^{1}=\psi_{\lambda}^{2}$, since $\mathcal{E}$ is bounded in $L^{1}(0, T)$.

Appendix A. Some technical lemmas. We state first the following Lemma. The (easy) proof is left to the reader.

Lemma A.1. Let $f \in H^{1 / 2}(0, T)$, and define:

$$
\partial^{1 / 2} f:=\frac{1}{\sqrt{\pi}} \frac{d}{d t} \int_{0}^{t} \frac{f(\tau)}{\sqrt{t-\tau}} d \tau, \quad 0 \leq t \leq T .
$$

Denoting by $E f$ the extension by zero outside $(0, T)$ and by $\widehat{f}$ its Fourier transform, we have the identities

$$
\begin{aligned}
\partial^{1 / 2} f & =\frac{1}{\sqrt{\pi}} \frac{d}{d t} E f *\left(\mathbb{1}_{\mathbb{R}^{+}} \frac{1}{\sqrt{\cdot}}\right), \quad t \in \mathbb{R}, \\
\widehat{\partial^{1 / 2} f}(\xi) & =e^{i \pi / 4}-\sqrt{\xi} \widehat{f}(\xi),
\end{aligned}
$$

where $\bar{V}$ stands for the complex square root with non-positive imaginary part and $\mathbb{1}_{\mathbb{R}^{+}}$ for the indicatrix function of $\mathbb{R}^{+}$. 
The second Lemma basically states that if a function $g(x)$ belong to $L^{2}(\mathbb{R})$, as well as $|x|^{1 / 4} g(x)$, and if $g$ is the Fourier transform of a function with a bounded support, then $g$ decays as $|x|^{-1 / 4}$.

Lemma A.2. Let $f$ be a function in $L^{2}(0, t)$. Denote by Ef its extension by zero outside $(0, t)$ and let $\widehat{f}$ be the Fourier transform of Ef. Assuming $k^{1 / 4} \widehat{f}(k) \in L^{2}\left(\mathbb{R}^{+}\right)$, we have, for any $a>0$, for $\xi \geq a$,

$$
|\widehat{f}(\xi)| \leq C\left((1+t) \xi^{-1 / 4}\left\|k^{1 / 4} \widehat{f}\right\|_{L^{2}\left(\mathbb{R}^{+}\right)}+\xi^{-1 / 2}\|\widehat{f}\|_{L^{2}(\mathbb{R})}\right),
$$

where $C$ depends on a.

Proof. The proof strongly relies on the fact that we compute the Fourier transform of a function with bounded support. From the definition, we have

$$
\widehat{f}(\xi)=\int_{0}^{t} f(s) e^{-i \xi s} d s=\widehat{\mathbb{1}_{[0, t]} E} f=(H * \hat{f})(\xi)
$$

where $H(\xi)=\widehat{\mathbb{1}_{[0, t]}}=(-i \xi)^{-1}\left(e^{-i \xi t}-1\right) . H$ satisfies

$$
|H(\xi)| \leq t, \quad \forall \xi \in \mathbb{R} ; \quad|H(\xi)| \leq \frac{2}{|\xi|}, \quad \forall|\xi|>0 .
$$

We assume that $\xi \geq a>0$. The convolution $H * \widehat{f}$ is then split into four terms, with $0<\beta<a$ :

$$
\begin{aligned}
\int_{\mathbb{R}} H(\xi-y) \widehat{f}(y) d y & =\int_{\mathbb{R}_{-}}+\int_{0}^{\xi-\beta}+\int_{\xi-\beta}^{\xi+\beta}+\int_{\xi+\beta}^{+\infty} \\
& =I_{1}+I_{2}+I_{3}+I_{4} .
\end{aligned}
$$

Having in mind that $\xi \geq a>0$, if follows readily from (A.2) that

$$
I_{1} \leq\|H(\xi+\cdot)\|_{L^{2}\left(\mathbb{R}^{+}\right)}\|\widehat{f}\|_{L^{2}(\mathbb{R})} \leq 2 \xi^{-1 / 2}\|\widehat{f}\|_{L^{2}(\mathbb{R})} .
$$

Concerning $I_{2}$, we have

$$
\begin{aligned}
I_{2} & \leq\left(\int_{0}^{\xi-\beta} \frac{H(\xi-y)^{2}}{y^{1 / 2}} d y\right)^{1 / 2}\left\|y^{1 / 4} \widehat{f}(y)\right\|_{L^{2}\left(\mathbb{R}^{+}\right)} \\
& \leq 2\left(\int_{0}^{\xi-\beta} \frac{d y}{|\xi-y|^{2} y^{1 / 2}}\right)^{1 / 2}\left\|y^{1 / 4} \widehat{f}(y)\right\|_{L^{2}\left(\mathbb{R}^{+}\right)} .
\end{aligned}
$$

Consider now an $\alpha$ such that $0<\alpha<1-\beta / a<1-\beta / \xi$ and $\alpha<a$. Then

$$
\begin{aligned}
\int_{0}^{\xi-\beta} \frac{d y}{|\xi-y|^{2} y^{1 / 2}} & =\frac{1}{\xi^{3 / 2}} \int_{0}^{1-\beta / \xi} \frac{d y}{|y-1|^{2} y^{1 / 2}} \\
& =\int_{0}^{\alpha}+\int_{\alpha}^{1-\beta / \xi} \\
& \leq \frac{1}{\xi^{3 / 2}} \frac{2 \alpha^{1 / 2}}{(1-\alpha)^{2}}+\frac{1}{\xi^{3 / 2}} \frac{1}{\alpha^{1 / 2}}\left(\frac{\xi}{\beta}+\frac{1}{1-\alpha}\right) \\
& =\mathcal{O}\left(\xi^{-1 / 2}\right) .
\end{aligned}
$$


Proceeding analogously, $I_{3}$ is easily estimated thanks to (A.2)

$$
\begin{aligned}
I_{3} & \leq t\left(\int_{\xi-\beta}^{\xi+\beta} \frac{d y}{\sqrt{y}}\right)^{1 / 2}\left\|y^{1 / 4} \widehat{f}(y)\right\|_{L^{2}\left(\mathbb{R}^{+}\right)} \\
& \leq \frac{\sqrt{2 \beta} t}{(\xi-\beta)^{1 / 4}}\left\|y^{1 / 4} \widehat{f}(y)\right\|_{L^{2}\left(\mathbb{R}^{+}\right)} .
\end{aligned}
$$

In the same way,

$$
\begin{aligned}
I_{4} & \leq 2\left(\int_{\xi+\beta}^{\infty} \frac{d y}{|\xi-y|^{2} y^{1 / 2}}\right)^{1 / 2}\left\|y^{1 / 4} \widehat{f}(y)\right\|_{L^{2}\left(\mathbb{R}^{+}\right)} \\
& \leq \frac{2}{\sqrt{\beta}(\xi+\beta)^{1 / 4}}\left\|y^{1 / 4} \widehat{f}(y)\right\|_{L^{2}\left(\mathbb{R}^{+}\right)}
\end{aligned}
$$

The proof is then ended by gathering the different estimates on $I_{1}, I_{2}, I_{3}$ and $I_{4}$.

REMARK A.3. The previous lemma can be generalized to functions of the form $g(s) f(s)$ where $f$ satisfies the hypothesis of the lemma and $g$ is a $\mathcal{C}^{1}([0, t])$ function. Indeed, in this case the convolution kernel $H$ satisfies, instead of (A.2),

$$
|H(\xi)| \leq C_{1}, \quad \forall \xi \in \mathbb{R} ; \quad|H(\xi)| \leq \frac{C_{2}}{|\xi|}, \quad \forall|\xi|>0,
$$

for two positive constants $C_{1}$ and $C_{2}$, so that the proof of the lemma still applies. The result is thus

$$
|\widehat{f g}(\xi)| \leq C\left((1+t) \xi^{-1 / 4}\left\|k^{1 / 4} \widehat{f}\right\|_{L^{2}\left(\mathbb{R}^{+}\right)}+\xi^{-1 / 2}\|\widehat{f}\|_{L^{2}(\mathbb{R})}\right),
$$

where the constant $C$ depends on $\|g\|_{\mathcal{C}^{1}([0, t])}$. This relation is used at the end of the proof of the energy estimate.

The last lemma is a reformulation of a standard trace theorem when considering flat boundaries. Notice that applying straightforwardly the existing results would have given

$$
\|\varphi\|_{L^{2}\left(\Gamma_{j}\right)} \leq C\|\varphi\|_{L^{2}\left(\Omega_{0}\right)}^{1 / 2-\alpha}\|\nabla \varphi\|_{L^{2}\left(\Omega_{0}\right)}^{1 / 2+\alpha}, \quad \alpha>0, \quad j=1, \ldots, n,
$$

while we have the following lemma:

Lemma A.1. Let $\varphi \in H^{1}\left(\Omega_{0}\right)$ where $\Omega_{0}$ is smooth (at least $\mathcal{C}^{1}$ ) and is defined in Section 2. Assume that the trace of $\varphi$ vanishes on $\Gamma_{0}$. Then there exists $C>0$ such that

$$
\|\varphi\|_{L^{2}\left(\Gamma_{j}\right)} \leq C\|\varphi\|_{L^{2}\left(\Omega_{0}\right)}^{1 / 2}\|\nabla \varphi\|_{L^{2}\left(\Omega_{0}\right)}^{1 / 2}, \quad j=1, \ldots, n .
$$

Proof. We assume that $\varphi$ is regular and proceed by density. Let us parametrize $\Omega_{0}$ by an orthogonal set of coordinates $x=\left(x^{\prime}, \eta\right)$ such that if $\eta=0$, then $x$ belongs to the plane into which $\Gamma_{j}$ is included. Such a construction is possible, since the interface $\Gamma_{j}$ is plane. Thus $\varphi\left(x^{\prime}, 0\right)$ is the trace of $\varphi$ on $\Gamma_{j}$ for $x^{\prime} \in \Gamma_{j}$. We have

$$
\left|\varphi\left(x^{\prime}, \eta\right)\right|^{2}=\left|\varphi\left(x^{\prime}, 0\right)\right|^{2}+2 \mathcal{R} e \int_{0}^{\eta} \bar{\varphi}\left(x^{\prime}, y\right) \frac{\partial \varphi\left(x^{\prime}, y\right)}{\partial y} d y .
$$


Then, the Cauchy-Schwarz inequality implies that

$$
\|\varphi\|_{L^{2}\left(\Gamma_{j}\right)}^{2} \leq\|\varphi\|_{L^{2}\left(\Omega_{0}\right)}^{2}+2\|\varphi\|_{L^{2}\left(\Omega_{0}\right)}\|\nabla \varphi\|_{L^{2}\left(\Omega_{0}\right)} .
$$

Finally, the Poincar ineqality allows to control $\|\varphi\|_{L^{2}\left(\Omega_{0}\right)}$ in terms of $\|\nabla \varphi\|_{L^{2}\left(\Omega_{0}\right)}$, and this concludes the proof.

Appendix B. Sketch of the regularization. The particular geometry and the different assumptions made on the data render the problem not straightforward to regularize. Indeed, the initial wave function is supposed to crucially solve a Schrdinger equation in each wave guide and the potentials have as well some important properties. We give here the main ideas about how this procedure can be pursued.

Let $\rho$ be a standard mollifier such that $0 \leq \rho \leq 1, \rho \in \mathcal{C}^{\infty}\left(\mathbb{R}^{d}\right),\|\rho\|_{L^{1}}=1$ and whose support is included in the unit ball centered at zero. We denote by $\rho^{\delta}:=\rho\left(\frac{x}{\delta}\right)$ and by $E$ an extension operator either from $(0, T) \Omega$ to $\mathbb{R}^{d+1}$ or from $\Omega$ to $\mathbb{R}^{d}$, see [1]. Let $V_{e} \in \mathcal{C}^{1}\left([0, T], L^{\infty}(\Omega)\right)$ be in the class of potentials introduced in Section 2.3, and define $V_{e}^{\delta}:=\rho^{\delta} *_{t}\left(\rho^{\delta} *_{x} E V_{e}\right)$, where we use a one-dimensional mollifier for the time variable. Then $V_{e}^{\delta} \in \mathcal{C}^{\infty}\left(\mathbb{R}^{d+1}\right)$ and $V_{e}^{\delta}$ converges strongly to $V_{e}$ in $\mathcal{C}^{1}\left([0, T], L^{p}(K)\right), p<\infty$, for any bounded set $K \subset \Omega$. It is assumed in each wave guide $\Omega_{j}$ (equipped with the set of coordinates $\left.\left(\xi_{j}, \eta_{j}\right)\right)$ that $V_{e}$ verifies $V_{e}(t, x)=V^{0}\left(\xi_{j}\right)+V_{j}(t)$, which is not true for $V_{e}^{\delta}$. Nevertheless, as soon as $\eta_{j} \geq \delta, V_{e}^{\delta}$ shares a similar structure, that is, $V_{e}^{\delta}(t, x)=$ $V^{0, \delta}\left(\xi_{j}\right)+V_{j}^{\delta}(t)$, where $V^{0, \delta}\left(\xi_{j}\right)=\int_{\mathbb{R}^{d-1}} \int_{-\delta}^{\delta} E V^{0}(z) \rho\left(\xi_{j}-z, y\right) d z d y$ and $V_{j}^{\delta}=\rho *_{t} E V_{j}$. This suggests to define a fixed parameter $\delta_{0}$, with $0<\delta<\delta_{0}$, such that the transparent boundary conditions are prescribed on $\left.\Gamma_{j}^{\delta_{0}}=\left\{\left(\eta_{j}, \xi_{j}\right) \in \Omega_{j}, \xi_{j} \in \Gamma_{j}, \eta_{j}=\delta_{0}\right)\right\}$. It is not possible to define them for $x \in \Omega_{j}, \eta_{j} \leq \delta$, since $V_{e}^{\delta}$ does not satisfy the adequate decomposition.

In the same way, let $\psi_{\lambda}^{0, \delta}:=\chi^{\delta} \rho^{\delta} *_{x}\left(E \psi_{\lambda}^{0}\right) \in \mathcal{C}^{\infty}(\Omega)$, where $\chi$ is a cut-off which ensures that $\psi_{\lambda}^{0, \delta}$ vanishes on the boundary $\partial \Omega$. $\psi_{\lambda}^{0, \delta}$ converges strongly to $\psi_{\lambda}^{0}$ in $H^{1}(K)$, for any bounded set $K \subset \Omega$. Let now $\chi_{m}^{0, j, \delta}$ be the solution to (2.1) with the regularized potential $V^{0, \delta}$ and $E_{m}^{j, \delta}$ be the associated eigenvalue. According to the regularity of the potential, $\chi_{m}^{0, j, \delta} \in \mathcal{C}^{\infty}\left(\Gamma_{j}\right)$. In each guide $\Omega_{j}, \psi_{\lambda}^{0}$ reads:

$$
\begin{aligned}
\psi_{\lambda}^{0}\left(\xi_{j}, \eta_{j}\right)=\sum_{m} & {\left[\chi_{m}^{0, j}\left(\xi_{j}\right) \delta_{m}^{m_{0}} \delta_{j}^{j_{0}} \exp \left(\frac{-i \eta_{j}}{\varepsilon} \sqrt[+]{2\left(E(\lambda)-E_{m}^{j}\right)}\right)\right.} \\
+ & \left.b_{m}^{j} \exp \left(\frac{i \eta_{j}}{\varepsilon} \sqrt[+]{2\left(E(\lambda)-E_{m}^{j}\right)}\right)\right] .
\end{aligned}
$$

This leads to the definition of

$$
\begin{aligned}
& \varphi_{\lambda}^{\delta}\left(\xi_{j}, \eta_{j}\right)=\sum_{m} {\left[\chi_{m}^{0, j, \delta}\left(\xi_{j}\right) \delta_{m}^{m_{0}} \delta_{j}^{j_{0}} \exp \left(\frac{-i \eta_{j}}{\varepsilon} \sqrt[+]{2\left(E(\lambda)-E_{m}^{j, \delta}\right)}\right)\right.} \\
&\left.+b_{m}^{j} \exp \left(\frac{i \eta_{j}}{\varepsilon} \sqrt[+]{2\left(E(\lambda)-E_{m}^{j, \delta}\right)}\right)\right],
\end{aligned}
$$

so that $\varphi_{\lambda}^{\delta} \in \mathcal{C}^{\infty}\left(\Omega_{j}\right)$ and $\varphi_{\lambda}^{\delta}$ converges strongly to $\psi_{\lambda}^{0}$ in $H^{1}(K)$, for any bounded set $K \subset \Omega_{j}$. In each wave guide, we thus have, for $\eta_{j} \geq 0$,

$$
-\frac{\varepsilon^{2}}{2} \Delta \varphi_{\lambda}^{\delta}+V^{0, \delta} \varphi_{\lambda}^{\delta}=E(\lambda) \varphi_{\lambda}^{\delta} .
$$

Let $\chi$ be another smooth cut-off function equal to one on $\Omega_{0}$ and whose support is included in the set $\left\{x \in \mathbb{R}^{d}, \eta_{j} \leq \delta_{0}, j=1, \ldots, n\right\}$, and let $\widetilde{\psi_{\lambda}^{0, \delta}}=\chi \psi_{\lambda}^{0, \delta}+(1-\chi) \varphi_{\lambda}^{\delta}$. We 
have that $\widetilde{\psi_{\lambda}^{0, \delta}} \in \mathcal{C}^{\infty}(\Omega)$, and $\widetilde{\psi_{\lambda}^{0, \delta}}$ converges strongly to $\psi_{\lambda}^{0}$ in $H^{1}(K)$, for any bounded set $K \subset \Omega$.

Consider now the following regularized Schrdinger-Poisson problem:

$$
\begin{gathered}
i \varepsilon \frac{\partial \psi_{\lambda}^{\delta}}{\partial t}=-\frac{\varepsilon^{2}}{2} \Delta \psi_{\lambda}^{\delta}+\left(V_{e}^{\delta}+V_{s}^{\delta}\right) \psi_{\lambda}^{\delta} ; \quad \psi_{\lambda}^{\delta}(t=0, \cdot)=\widetilde{\psi_{\lambda}^{0, \delta}} ; \quad x \in \Omega \\
-\Delta V_{s}^{\delta}=\int_{\Lambda}\left|\psi_{\lambda}^{\delta}\right|^{2} d \mu(\lambda) ; \quad x \in \Omega_{0} ;\left.\quad V_{s}^{\delta}\right|_{\partial \Omega_{0}}=0
\end{gathered}
$$

Define as well:

$$
\psi_{\lambda}^{p w, \delta}=\widetilde{\psi_{\lambda}^{0, \delta}} \sum_{j=1}^{n} \mu_{j} \theta_{\lambda}^{j, \delta} ; \quad \theta_{\lambda}^{j, \delta}(t)=\exp \left(-\frac{i}{\varepsilon} \int_{0}^{t}\left(E(\lambda)+V_{j}^{\delta}(s)\right) d s\right),
$$

where $\left(\mu_{j}\right)_{j}$ is the partition of unity introduced in Section 2.1. The previous construction of $\widetilde{\psi_{\lambda}^{0, \delta}}$ and $V_{e}^{\delta}$ insures that

$$
i \varepsilon \frac{\partial \psi_{\lambda}^{p w, \delta}}{\partial t}=-\frac{\varepsilon^{2}}{2} \Delta \psi_{\lambda}^{p w, \delta}+\left(V_{e}^{\delta}+V_{s}^{\delta}\right) \psi_{\lambda}^{p w, \delta}, \quad x \in \Omega_{j}, \quad \eta_{j} \geq \delta_{0},
$$

so that Theorem 3.1 applies and provides a unique strong solution $\left(\psi_{\lambda}^{\delta}, V_{s}^{\delta}\right)$. The $\mathcal{C}^{\infty}$ regularity is easily deduced from that of the data. Moreover, $\psi_{\lambda}^{\delta}$ satisfies:

$$
\frac{\partial}{\partial \eta_{j}}\left(\psi_{\lambda}^{\delta}-\psi_{\lambda}^{p w, \delta}\right)=-\frac{e^{-i \pi / 4}}{\sqrt{\varepsilon}} \mathbb{D}_{j, \delta}^{1 / 2}\left(\psi_{\lambda}^{\delta}-\psi_{\lambda}^{p w, \delta}\right) ; \quad x \in \Gamma_{j}^{\delta_{0}} .
$$

The definition of $\mathbb{D}_{j, \delta}^{1 / 2}$ is the same as $(3.5), \chi_{m}^{0, j}$ being replaced by $\chi_{m}^{0, j, \delta}$ so that we have (4.1)-(4.3).

For the sake of clarity of the paper and without loss of generality, we abusively decided to set the boundary conditions in the proof of the theorem on the interfaces $\Gamma_{j}$ instead of $\Gamma_{j}^{\delta_{0}}$.

Acknowledgments. The author would like to thank Naoufel Ben Abdallah for many discussions about the subject.

\section{REFERENCES}

[1] R.A. Adams, Sobolev Spaces, Academic Press, NY, San Francisco, London, 1975.

$[2]$ D. Ahluwalia and J. Keller, Exact and asymptotic representation of the sound field in a stratified ocean, Wave Propagation in Underwater Acoustics, Lecture Notes in Physics, Springer, 70, 14-85, 1977.

[3] X. Antoine and C. Besse, Construction, structure and asymptotic approximations of a microdifferential transparent boundary condition for the linear Schrdinger equation, J. Maths. Pures Appl., 9, 701-738, 2001.

[4] A. Arnold, Mathematical concept of open quantum boundary conditions, Transp. Theory. Stat. Phys., 6, 561-584, 2001.

[5] A. Arnold, M. Ehrhardt and I.M. Sofronov, Approximation, stability and fast calculations of non-local boundary conditions for the Schrdinger equation, Commun. Math. Sci., 1, 501556, 2003.

[6] G. Bal, G. Papanicolaou and L. Ryzhik, Radiative transport limit for the random Schrdinger equation, Nonlinearity, 15, 513-529, 2002.

[7] C. Bardos, F. Golse and N. Mauser, Weak coupling limit of the N-particle Schrdinger equation, Methods Appl. Anal., 7, 275-293, 2000. 
[8] G. Bastard, Wave Mechanics Applied to Semiconductor Heterostructures, Halsted Press, 1988.

[9] N. Ben Abdallah, A hybrid kinetic-quantum model for stationary electron transport in a resonant tunneling diode, J. Stat. Phys., 90, 627-662, 1998.

[10] N. Ben Abdallah, On a muldidimensional Schrdinger-Poisson scattering model for semiconductors, J. Math. Phys., 41, 4241-4261, 2000.

[11] N. Ben Abdallah, P. Degond and P.A. Markowich, On a one-dimensional Schrdinger-Poisson scattering model, ZAMP, 48, 135-155, 1997.

[12] N. Ben Abdallah, F. Mhats and O. Pinaud, On a open transient Schrdinger-Poisson system, Math. Meth. Mod. App. Sci., 15, 667-688, 2005.

[13] J. Benamou, F. Castella, T. Katsaounis and B. Perthame, High frequency limit in the Helmholtz equation, Rev. Mat. Iberoamericana, 18, 187-209, 2002.

[14] P. Degond and A. El Ayyadi, A coupled Schrdinger drift-diffusion model for quantum semiconductor device simulations, J. Comput. Phys., 181, 222-259, 2002.

[15] M. Ehrhardt and R.E. Mickens, Solutions to the discrete Airy equation: application to parabolic equation calculations, J. Comput. Appl. Math., 172, 183-206, 2004.

[16] B. Engquist and A. Majda, Absorbing boundary conditions for the numerical simulation of waves, Math. Comput., 31, 629-651, 1971.

[17] L. Erds and H.T. Yau, Linear Boltzmann equation as the weak coupling limit of a random Schrdinger Equation, Comm. Pure Appl. Math., 53(6), 667-735, 2000.

[18] P. Grard, P.A. Markowich, N.J. Mauser and F. Poupaud, Homogenization limits and Wigner transforms, Comm. Pure Appl. Math., 50, 323-380, 1997.

[19] J. Ginibre and G. Velo, On a class of nonlinear Schrdinger equations with non-local interaction, Math. Z., 170, 109-136, 1980.

[20] R. Gorenflo and S. Vessela, Abel Integral Equations, Springer, NY, Berlin, 1991.

[21] P.L. Lions and T. Paul, Sur les mesures de Wigner, Rev. Mat. Iberoamericana, 9, 553-618, 1993.

[22] P.A. Markowich and N.J. Mauser, The classical limit of a self-consistent quantum-Vlasov equation in 3-D, Math. Meth. Mod. Appl. Sci., 3, 109-124, 1993.

[23] F. Nier, Schrdinger-Poisson systems in dimension $d \leq 3$ : the whole space case, Proc. Roy. Soc. Edinburgh Section A, 23, 489-510, 1993.

[24] F. Nier, The dynamics of some open systems with short-range non-linearity, Nonlinearity, 11, 27-72, 1998.

[25] A. Pazy, Semigroups of Linear Operators and Applications to Partial Differential Equations, Springer, NY, Berlin, 1983.

[26] O. Pinaud, in preparation.

[27] E. Polizzi and N. Ben Abdallah, Subband decomposition approach for the simulation of quantum electron transport in nanostructures, J. Comput. Physics, 202, 150-180, 2004.

[28] L. Ryzhik, G. Papanicolaou and J.B. Keller, Transport equations for elastic and other waves in random media, Wave Motion, 24, 327-370, 1996.

[29] S. Samko, A. Kilbas and O. Marichev, Fractional Integrals and Derivatives, Gordon and Breach, 1993.

[30] F. Tappert, The parabolic approximation method, Wave Propagation in Underwater Acoustics, Lecture Notes in Physics, Springer, 70, 224-287, 1977.

[31] R. Temam, Navier-Stokes Equations: Theory and Numerical Analysis, North Holland, 1984.

[32] E. Wigner, On the quantum correction for thermodynamic equilibrium, Phys. Rev., 40, 742-749, 1932. 